\title{
Radially excited rotating black holes in Einstein-Maxwell-Chern-Simons theory
}

\author{
Jose Luis Blázquez-Salcedo, ${ }^{1}$ Jutta Kunz, ${ }^{1}$ Francisco Navarro-Lérida, ${ }^{2}$ and Eugen Radu ${ }^{3}$ \\ ${ }^{1}$ Institut für Physik, Universität Oldenburg Postfach 2503, D-26111 Oldenburg, Germany \\ ${ }^{2}$ Departamento de Física Atómica, Molecular y Nuclear, Ciencias Físicas \\ Universidad Complutense de Madrid, E-28040 Madrid, Spain \\ ${ }^{3}$ Departamento de Física da Universidade de Aveiro and I3N Campus de Santiago, \\ 3810-183 Aveiro, Portugal \\ (Received 26 June 2015; published 17 August 2015)
}

\begin{abstract}
Rotating black holes in Einstein-Maxwell-Chern-Simons theory possess remarkable features when the Chern-Simons coupling constant reaches a critical value. Representing single asymptotically flat black holes with horizons of spherical topology, they exhibit nonuniqueness. In particular, there even exist extremal and nonextremal black holes with the same sets of global charges. Both extremal and nonextremal black holes form sequences of radially excited solutions that can be labeled by the node number of the magnetic gauge potential function. The extremal Reissner-Nordström solution is no longer always located on the boundary of the domain of existence of these black holes, nor does it remain the single extremal solution with vanishing angular momentum. Instead a whole sequence of rotating extremal $J=0$ solutions is present, whose mass converges towards the mass of the Reissner-Nordström solution. These radially excited extremal solutions are all associated with the same near horizon solution. Moreover, there are near horizon solutions that are not realized as global solutions.
\end{abstract}

DOI: 10.1103/PhysRevD.92.044025

PACS numbers: 04.40.Nr, 04.20.Jb, 04.50.-h

\section{INTRODUCTION}

In four spacetime dimensions vacuum black holes and black holes in the presence of an electromagnetic field possess very remarkable properties, as formulated by a number of important theorems. The black hole uniqueness theorem, for instance, states that asymptotically flat nondegenerate electrovac black holes are uniquely described by their global charges: the mass, the angular momentum and the electric (and magnetic) charge [1-4].

When going to higher dimensions, the Schwarzschild solutions and the Reissner-Nordström (RN) solutions possess simple generalizations, while the Kerr solutions are generalized by the family of Myers-Perry (MP) solutions [5,6]. In $D$ dimensions, these solutions possess $N=$ $\lfloor(D-1) / 2\rfloor$ independent spatial planes and thus also $N$ independent angular momenta. In five dimensions both angular momenta are bounded for a given mass, a feature which does not hold for $D>5$.

The higher-dimensional generalizations of the KerrNewman solutions are not known in closed form. However, they have been studied perturbatively [7-14] and numerically [15-18]. In odd dimensions, when all angular momenta possess equal magnitude, the angular coordinates factorize, leading to substantial simplifications of the perturbative and numerical studies. Moreover, for extremal black holes the near-horizon solutions have been investigated $[18,19]$. In the case of black hole solutions with equal magnitude angular momenta in odd dimensions, there are two branches of near-horizon solutions, the MP branch and the RN branch, intersecting at a critical point.
Interestingly, for both branches, only a part of the nearhorizon solutions also correspond to global solutions; thus not all Einstein-Maxwell (EM) near-horizon solutions have global counterparts.

In odd dimensions, a Chern-Simons (CS) term can be added to the action, breaking the charge reversal symmetry for even $N=(D-1) / 2$. The resulting Einstein-MaxwellChern-Simons (EMCS) theories possess intriguing sets of black hole solutions [20-25]. In five dimensions for the special case of the CS coupling constant $\lambda=\lambda_{\mathrm{SG}}$, as obtained for minimal supergravity, the general set of charged rotating black hole solutions is known in closed form [22].

Carrying opposite angular momenta, the BreckenridgeMyers-Peet-Vafa (BMPV) [20] black holes represent a subset of these solutions. Emerging from one of the charge symmetric extremal RN solutions, they form a branch of stationary extremal black hole solutions with vanishing horizon angular velocities. The magnitude of their (equal magnitude) angular momenta can be increased, while keeping their mass and charge fixed, until a singular solution with vanishing area is encountered. Recall that in four dimensions stationary EM black holes with a nonrotating horizon are static. It is the presence of the CS term, which allows for nonstatic black holes with vanishing horizon angular momenta. Here the frame dragging effects at the inside and outside of the horizon precisely cancel at the horizon in the case of the BMPV black holes [26].

When the CS coupling constant $\lambda$ is increased beyond its supergravity value, the set of black hole solutions acquires interesting new features. First, for $\lambda_{\mathrm{SG}} \leq \lambda<2 \lambda_{\mathrm{SG}}$, 
counterrotation sets in; i.e., within a certain region of the domain of existence of the solutions, the horizon angular velocity and the angular momentum have opposite signs $[23,24]$. As a consequence, extremal static black holes can become unstable with respect to rotation. Indeed, for fixed electric charge the mass can decrease with increasing magnitude of the angular momentum. Thus supersymmetry marks the borderline between stability and instability for EMCS black holes $[23,26]$.

Next, for $\lambda>2 \lambda_{\mathrm{SG}}$, the extremal RN solution remains part of the boundary of the domain of existence only for one sign of the charge. For the other sign of the charge, the extremal RN solution resides inside the boundary of the domain of existence. As a boundary solution with vanishing angular momentum the static RN solution is now replaced by a set of two stationary solutions, whose global angular momenta vanish [23]. Thus EMCS theory allows for black holes with rotating horizon but vanishing angular momenta, where the contributions to the global angular momenta precisely balance.

These two $J=0$ rotating extremal solutions form only the lowest mass solutions of a whole sequence of excited $J=0$ rotating extremal solutions, which possess an increasing number of radial nodes in one of the metric and one of the gauge field functions [25]. For a fixed value of the charge, the mass of this sequence of solutions converges towards the mass of the corresponding extremal RN black hole. Since these excited extremal black hole solutions are located inside the domain of existence these solutions represent a new type of violation of uniqueness: there are extremal and nonextremal black holes with the same sets of global charges [25]. The violation of uniqueness among nonextremal black holes is of course also present [23].

When the full sets of extremal solutions are considered, an intricate web of branches arises. These branches of global black hole solutions can be compared with the branches of near-horizon solutions. The structure of the latter is much simpler. Comparison of the two sets shows that there are near-horizon solutions, which correspond to (i) no global solutions, (ii) exactly one global solution, or (iii) more that one global solution [25].

Here we give a detailed account of the astounding properties of EMCS black hole solutions in five dimensions, treating the CS coupling constant as a free parameter, a task which, to our knowledge, has not been yet considered in the literature. Special attention will be paid to extremal solutions, although some properties of nonextremal configurations will be discussed as well. The paper is organized as follows: in Sec. II we present the action, the Ansätze and the charges. We discuss the near-horizon solutions in Sec. III, where the near-horizon formalism must be employed with care because of the presence of the CS term. We present our numerical procedure for the global solutions in Sec. IV, discuss the boundary conditions and provide expansions for the functions. Our numerical results together with a comparison of global and near-horizon solutions are given in Sec. V. We end with a brief conclusion and outlook in Sec. VI.

\section{ACTION, ANSÄTZE AND CHARGES}

We focus our study on black holes in EMCS theory. Here we briefly review the action and the general set of equations of motion. We then present the appropriate Ansätze to obtain rotating black holes with equal magnitude angular momenta. Next we recall the general formulas for the global charges and the horizon properties of these black holes, as well as their scaling symmetry.

\section{A. Einstein-Maxwell-Chern-Simons action}

The action of EMCS theory in five dimensions reads

$$
\begin{aligned}
I= & \frac{1}{16 \pi G_{5}} \int d^{5} x\left[\sqrt{-g}\left(R-\frac{1}{4} F_{\mu \nu} F^{\mu \nu}\right)\right. \\
& \left.-\frac{\lambda}{12 \sqrt{3}} \varepsilon^{\mu \nu \alpha \beta \gamma} A_{\mu} F_{\nu \alpha} F_{\beta \gamma}\right],
\end{aligned}
$$

where $R$ is the curvature scalar, $G_{5}$ is Newton's constant in five dimensions, $A_{\mu}$ is the gauge potential with field strength tensor $F_{\mu \nu}=\partial_{\mu} A_{\nu}-\partial_{\nu} A_{\mu}$, and $\lambda$ is the CS coupling constant. In the following we employ units such that $16 \pi G_{5}=1$. Note that for $\lambda=\lambda_{\mathrm{SG}}=1$ the action corresponds to the bosonic sector of minimal supergravity. For $\lambda=\lambda_{\mathrm{EM}}=0$ the action corresponds to EinsteinMaxwell theory.

Variation of the action with respect to the metric leads to the Einstein equations

$$
G_{\mu \nu}=\frac{1}{2} T_{\mu \nu},
$$

where the stress-energy tensor is given by

$$
T_{\mu \nu}=F_{\mu \rho} F_{\nu}{ }^{\rho}-\frac{1}{4} g_{\mu \nu} F_{\rho \sigma} F^{\rho \sigma} .
$$

Variation with respect to the gauge potential leads to the Maxwell-Chern-Simons equations

$$
\nabla_{\nu} F^{\mu \nu}+\frac{\lambda}{4 \sqrt{3}} \varepsilon^{\mu \nu \alpha \beta \gamma} F_{\nu \alpha} F_{\beta \gamma}=0 .
$$

\section{B. Ansätze}

We consider stationary black holes, which represent generalizations of the five-dimensional MP solutions [5] to EMCS theory. Hence these black holes possess a spherical horizon topology. Their Killing vectors are

$$
\xi \equiv \partial_{t}, \quad \eta_{(1)} \equiv \partial_{\varphi_{1}}, \quad \eta_{(2)} \equiv \partial_{\varphi_{2}},
$$


with $t$ the time coordinate and $\varphi_{1,2}$ angular directions. With each azimuthal symmetry an angular momentum $J_{(k)}$, $k=1,2$, is associated. In general, both angular momenta are independent.

Here we restrict to the case where both angular momenta have equal magnitude, $\left|J_{(1)}\right|=\left|J_{(2)}\right|=|J|$. The spacetime then becomes a cohomogeneity-1 manifold, with an enhancement of the isometry group from $R_{t} \times U(1)^{2}$ to $R_{t} \times U(2)$. Hence the angular dependence of the metric and the gauge potential can be explicitly given. Then the metric may be parametrized by the Ansatz

$$
\begin{aligned}
d s^{2}= & -F_{0}(r) d t^{2}+F_{1}(r) d r^{2}+F_{2}(r) d \theta^{2} \\
& +F_{3}(r) \sin ^{2} \theta\left(\varepsilon_{1} d \varphi_{1}-W(r) d t\right)^{2} \\
& +F_{3}(r) \cos ^{2} \theta\left(\varepsilon_{2} d \varphi_{2}-W(r) d t\right)^{2} \\
& +\left(F_{2}(r)-F_{3}(r)\right) \sin ^{2} \theta \cos ^{2} \theta\left(\varepsilon_{1} d \varphi_{1}-\varepsilon_{2} d \varphi_{2}\right)^{2},
\end{aligned}
$$

where $\theta \in[0, \pi / 2], \varphi_{1} \in[0,2 \pi]$ and $\varphi_{2} \in[0,2 \pi]$, and $\varepsilon_{k}=$ \pm 1 denotes the sense of rotation in the $k$ th orthogonal plane of rotation. Note also that the line element Eq. (6) still possesses a residual metric gauge freedom.

The corresponding Ansatz for the gauge potential is given by

$$
A_{\mu} d x^{\mu}=a_{0}(r) d t+a_{\varphi}(r)\left(\sin ^{2} \theta \varepsilon_{1} d \varphi_{1}+\cos ^{2} \theta \varepsilon_{2} d \varphi_{2}\right) .
$$

\section{Known solutions and parametrization used in the numerics}

For the general family of EMCS solutions within the above Ansätze, only the following special cases are known in closed form:

(i) Vacuum rotating black holes (Myers-Perry solution). This solution is valid for every value of $\lambda$, since the Maxwell and the CS terms vanish identically. The functions which enter the line element Eq. (6) are

$$
\begin{aligned}
& F_{0}(r)=\frac{1-\frac{M}{6 \pi^{2} r^{2}}+\frac{3 J^{2}}{8 \pi^{2} M r^{4}}}{1+\frac{3 J^{2}}{8 \pi^{2} M r^{4}}}, \\
& F_{1}(r)=\frac{1}{1+\frac{3 J^{2}}{8 \pi^{2} M r^{4}}, \quad F_{2}(r)=r^{2},} \\
& F_{3}(r)=r^{2}\left(\frac{3 J^{2}}{8 \pi^{2} M r^{4}}\right), \\
& W(r)=\frac{J}{4 \pi^{2} r^{4}\left(1+\frac{3 J^{2}}{8 \pi^{2} M r^{4}}\right)},
\end{aligned}
$$

while $a_{\varphi}(r)=a_{0}(r)=0$. Also, $M$ and $J$ are the mass and the angular momentum of the solutions. (ii) Charged static black holes (Reissner-Nordström solution). Again this solution is valid for every value of $\lambda$. In the static case, the magnetic component of the gauge field vanishes, $a_{\varphi}(r)=0$, and the CS term does not contribute. The expressions of the other functions which enter the solution are

$$
\begin{aligned}
& F_{0}(r)=\frac{1}{F_{1}(r)}=1-\frac{M}{6 \pi^{2} r^{2}}+\frac{Q^{2}}{48 \pi^{2} r^{4}}, \\
& F_{2}(r)=F_{3}(r)=r^{2}, \\
& W(r)=0, \quad \text { and } \quad a_{0}(r)=\frac{Q}{4 \pi^{2} r^{2}},
\end{aligned}
$$

with $Q$ the electric charge.

(iii) The general set of charged rotating solutions for $\lambda=$ $\lambda_{\mathrm{SG}}=1$ [22] with the extremal BMPV black holes [20] as a special case. These black holes have

$$
\begin{aligned}
& F_{0}(r)=\frac{1-\frac{2(p-q)}{r^{2}}+\frac{2 j^{2} p+q^{2}}{r^{4}}}{1+\frac{2 j^{2} p}{r^{4}}-\frac{j^{2} q^{2}}{r^{6}}}, \\
& F_{1}(r)=\frac{1}{1-\frac{2(p-q)}{r^{2}}+\frac{2 j^{2} p+q^{2}}{r^{4}}}, \quad F_{2}(r)=r^{2}, \\
& F_{3}(r)=\left(1+\frac{2 j^{2} p}{r^{4}}-\frac{j^{2} q^{2}}{r^{6}}\right) r^{2}, \\
& W(r)=\frac{2 p-q-\frac{q^{2}}{r^{2}}}{1+\frac{2 j^{2} p}{r^{4}}-\frac{j^{2} q^{2}}{r^{6}}}, \quad \text { and } \\
& r^{4}(r)=-\frac{\sqrt{3} j q}{r^{2}}, \quad a_{0}(r)=\frac{\sqrt{3} q}{r^{2}},
\end{aligned}
$$

with $p=\frac{M+\sqrt{3} Q}{12 \pi^{2}}, j=\frac{3 J}{2 M+\sqrt{3} Q}, q=\frac{Q}{4 \sqrt{3} \pi^{2}}$.

In the numerics, we have found it useful to use quasiisotropic coordinates by choosing a metric gauge with $F_{2}(r)=F_{1}(r) r^{2}$ and then to take $F_{0}(r)=f(r)$, $F_{1}(r)=\frac{m(r)}{f(r)}, F_{3}(r)=\frac{n(r)}{f(r)} r^{2}, W(r)=\frac{w(r)}{r}$. This results in the following expression of the line element in terms of four unknown functions,

$$
\begin{aligned}
d s^{2}= & -f(r) d t^{2}+\frac{m(r)}{f(r)}\left(d r^{2}+r^{2} d \theta^{2}\right) \\
& +\frac{n(r)}{f(r)} r^{2} \sin ^{2} \theta\left(\varepsilon_{1} d \varphi_{1}-\frac{\omega(r)}{r} d t\right)^{2} \\
& +\frac{n(r)}{f(r)} r^{2} \cos ^{2} \theta\left(\varepsilon_{2} d \varphi_{2}-\frac{\omega(r)}{r} d t\right)^{2} \\
& +\frac{m(r)-n(r)}{f(r)} r^{2} \sin ^{2} \theta \cos ^{2} \theta\left(\varepsilon_{1} d \varphi_{1}-\varepsilon_{2} d \varphi_{2}\right)^{2},
\end{aligned}
$$

originally proposed in $[15,16]$. 


\section{Global charges}

We here consider stationary asymptotically flat black holes. Their total mass $M$ and angular momentum $J_{(k)}$ can be obtained directly from the Komar expressions associated with the Killing vector fields

$$
\begin{aligned}
& M=-\frac{3}{2} \int_{S_{\infty}^{3}} \alpha, \\
& J_{(k)}=\int_{S_{\infty}^{3}} \beta_{(k)},
\end{aligned}
$$

where $\quad \alpha_{\mu_{1} \mu_{2} \mu_{3}} \equiv \epsilon_{\mu_{1} \mu_{2} \mu_{3} \rho \sigma} \nabla^{\rho} \xi^{\sigma}$, and $\quad \beta_{(k) \mu_{1} \mu_{2} \mu_{3}} \equiv \epsilon_{\mu_{1} \mu_{2} \mu_{3} \rho \sigma}$ $\nabla^{\rho} \eta_{(k)}^{\sigma}$. For equal-magnitude angular momenta $J_{(k)}=$ $\varepsilon_{k} J, k=1,2$.

The electric charge $Q$ is given by

$$
Q=-\frac{1}{2} \int_{S_{\infty}^{3}}\left(\tilde{F}+\frac{\lambda}{\sqrt{3}} A \wedge F\right)=-\frac{1}{2} \int_{S_{\infty}^{3}} \tilde{F},
$$

where $\tilde{F}_{\mu_{1} \mu_{2} \mu_{3}} \equiv \epsilon_{\mu_{1} \mu_{2} \mu_{3} \rho \sigma} F^{\rho \sigma}$. The magnetic moment $\mu_{\text {mag }}$ is determined from the asymptotic expansion of the gauge potential $a_{\varphi}(r)$; see Sec. IV B. The gyromagnetic ratio $g$ is then obtained from the magnetic moment $\mu_{\mathrm{mag}}$ via

$$
\mu_{\mathrm{mag}}=g \frac{Q J}{2 M} .
$$

\section{E. Horizon properties}

The black hole horizon is located at $r=r_{\mathrm{H}}$ and rotates with angular velocity $\Omega_{\mathrm{H}}$. It is a Killing horizon, where the Killing vector $\zeta=\partial_{t}+\Omega_{\mathrm{H}}\left(\varepsilon_{1} \partial_{\varphi_{1}}+\varepsilon_{2} \partial_{\varphi_{2}}\right)$ becomes null and orthogonal to the other Killing vectors,

$$
\begin{aligned}
\left.\left(\zeta^{2}\right)\right|_{\mathcal{H}} & =0, & & \left.\left(\zeta \cdot \partial_{t}\right)\right|_{\mathcal{H}}=0, \\
\left.\left(\zeta \cdot \partial_{\varphi_{1}}\right)\right|_{\mathcal{H}} & =0, & & \left.\left(\zeta \cdot \partial_{\varphi_{2}}\right)\right|_{\mathcal{H}}=0 .
\end{aligned}
$$

Note that these expressions impose conditions on the metric functions.

In particular, for the parametrization Eq. (11) employed in the numerics, the metric function $f(r)$ must vanish at the horizon,

$$
f\left(r_{\mathrm{H}}\right)=0,
$$

while the horizon angular velocity is given by

$$
\Omega_{\mathrm{H}}=\frac{\omega\left(r_{\mathrm{H}}\right)}{r_{\mathrm{H}}} .
$$

We focus our study on extremal black holes. In quasiisotropic coordinates, the event horizon of extremal black holes is given by $r_{\mathrm{H}}=0$. Note that in this case, the horizon angular velocity is obtained from

$$
\Omega_{\mathrm{H}}=\lim _{r_{\mathrm{H}} \rightarrow 0} \frac{\omega\left(r_{\mathrm{H}}\right)}{r_{\mathrm{H}}}=\left.\omega^{\prime}(r)\right|_{\mathcal{H}} .
$$

The area of the horizon $A_{\mathrm{H}}$ is given by

$$
A_{\mathrm{H}}=\int_{\mathcal{H}} \sqrt{\left|g^{(3)}\right|}=r_{\mathrm{H}}^{3} A\left(S^{3}\right) \lim _{r \rightarrow r_{\mathrm{H}}} \sqrt{\frac{m^{2} n}{f^{3}}},
$$

and the surface gravity $\kappa$ reads

$$
\kappa^{2}=-\left.\frac{1}{2}(\nabla \zeta)^{2}\right|_{\mathcal{H}}=\lim _{r \rightarrow r_{\mathrm{H}}} \frac{f}{\left(r-r_{\mathrm{H}}\right) \sqrt{m}} \cdot
$$

For extremal black holes the surface gravity vanishes, $\kappa=0$.

The horizon mass $M_{\mathrm{H}}$ and horizon angular momenta $J_{\mathrm{H}(k)}$ are given by

$$
\begin{aligned}
& M_{\mathrm{H}}=-\frac{3}{2} \int_{\mathcal{H}} \alpha, \\
& J_{\mathrm{H}(k)}=\int_{\mathcal{H}} \beta_{(k)} .
\end{aligned}
$$

For equal-magnitude angular momenta $J_{\mathrm{H}(k)}=\varepsilon_{k} J_{\mathrm{H}}$, $k=1,2$.

The electric charge $Q$ can also be determined at the horizon,

$$
Q=-\frac{1}{2} \int_{\mathcal{H}}\left(\tilde{F}+\frac{\lambda}{\sqrt{3}} A \wedge F\right)
$$

The horizon electrostatic potential $\Phi_{\mathrm{H}}$ is defined by

$$
\Phi_{\mathrm{H}}=\left.\zeta^{\mu} A_{\mu}\right|_{\mathcal{H}}=\left.\left(a_{0}+\Omega_{\mathrm{H}} a_{\varphi}\right)\right|_{\mathcal{H}}
$$

Note that $\Phi_{\mathrm{H}}$ is constant at the horizon.

\section{F. Scaling symmetry}

The EMCS solutions have the following scaling symmetry [23] (with $\tau>0$ an arbitrary parameter):

$$
\begin{aligned}
& \tilde{M}=\tau^{2} M, \quad \tilde{J}_{i}=\tau^{3} J_{i}, \quad \tilde{r}_{\mathrm{H}}=\tau r_{\mathrm{H}}, \\
& \tilde{\Omega}_{\mathrm{H}}=\Omega_{\mathrm{H}} / \tau, \quad \tilde{\kappa}=\kappa / \tau,
\end{aligned}
$$

etc. Let us therefore introduce scaled quantities, where we scale with respect to appropriate powers of the mass. These scaled quantities include the scaled angular momentum $j=J / M^{3 / 2}$, the scaled charge $q=Q / M$, the scaled area $a_{\mathrm{H}}=A_{\mathrm{H}} / M^{3 / 2}$, the scaled surface gravity $\bar{\kappa}=\kappa M^{1 / 2}$, and the scaled horizon angular velocity $\bar{\Omega}_{\mathrm{H}}=\Omega_{\mathrm{H}} M^{1 / 2}$. 


\section{G. Smarr formula}

The Smarr mass formula for EMCS black holes with two equal-magnitude angular momenta reads [26]

$$
M=3 \kappa A_{\mathrm{H}}+3 \Omega_{\mathrm{H}} J+\Phi_{\mathrm{H}} Q,
$$

or in terms of scaled quantities

$$
1=3 \bar{\kappa} a_{\mathrm{H}}+3 \bar{\Omega}_{\mathrm{H}} j+\Phi_{\mathrm{H}} q .
$$

Note that in the extremal case, the surface gravity vanishes. Moreover, in the extremal case

$$
M_{\mathrm{H}}=3 \Omega_{\mathrm{H}} J_{\mathrm{H}} \cdot
$$

EMCS black holes satisfy the first law [26],

$$
d M=2 \kappa d A_{\mathrm{H}}+2 \Omega_{\mathrm{H}} d J+\Phi_{\mathrm{H}} d Q .
$$

\section{EXTREMAL CONFIGURATIONS: NEAR- HORIZON SOLUTIONS}

Before analyzing the numerical solutions, some analytical understanding of the properties of extremal EMCS black holes can be achieved by deriving the near-horizon solutions in the entropy function formalism [27-29]. Employing this formalism we obtain semianalytic expressions for the entropy as a function of the electric charge and the angular momentum. We note, however, that the near horizon formalism needs special care, when it is employed in the presence of a CS term, as discussed extensively in the literature (see e.g. [30]).

In the following we first review the general entropy function formalism in the presence of a CS term. Subsequently, we apply the formalism to construct the near-horizon solutions of the extremal black holes in (i) pure Einstein-Maxwell theory, (ii) the bosonic sector of $D=5$ supergravity, and (iii) EMCS theory with general CS coupling constant $\lambda$.

\section{A. Entropy function formalism}

To apply the entropy function formalism to obtain the near-horizon geometry of extremal EMCS solutions, we make use of the Ansatz

$$
\begin{aligned}
d s^{2}= & v_{1}\left(\frac{d r^{2}}{r^{2}}-r^{2} d t^{2}\right) \\
& +v_{2}\left[4 d \theta^{2}+\sin ^{2} 2 \theta\left(\varepsilon_{2} d \varphi_{2}-\varepsilon_{1} d \varphi_{1}\right)^{2}\right] \\
& +v_{2} \eta\left[\varepsilon_{1} d \varphi_{1}+\varepsilon_{2} d \varphi_{2}\right. \\
& \left.+\cos 2 \theta\left(\varepsilon_{2} d \varphi_{2}-\varepsilon_{1} d \varphi_{1}\right)-\alpha r d t\right]^{2}
\end{aligned}
$$

Note the shift of the radial coordinate $r \rightarrow r-r_{\mathrm{H}}$. Thus the horizon is located at $r=0$.

For the gauge potential we employ the Ansatz

$$
A=-(\rho+p \alpha) r d t+2 p\left(\sin ^{2} \theta \varepsilon_{1} d \varphi_{1}+\cos ^{2} \theta \varepsilon_{2} d \varphi_{2}\right) .
$$

Here the parameters $v_{1}, v_{2}, \eta, \alpha, \rho$ and $p$ are constants, which satisfy a set of algebraic relations, that follow within the near-horizon formalism [27-29].

In the near-horizon formalism, the entropy is obtained from the extremum of the entropy function

$$
S=2 \pi(2 \alpha J+\rho \hat{q}-h),
$$

where $h$ is an action functional, that depends on the constants of the near horizon Ansatz,

$$
\begin{aligned}
h\left(\alpha, v_{1}, v_{2}, \eta, p, \rho\right)= & \int d \theta d \varphi_{1} d \varphi_{2} \sqrt{-g} \mathcal{L} \\
= & \frac{8 \pi^{2}}{9 v_{1} \sqrt{v_{2}}}\left(9 v_{2}^{3} \eta^{3 / 2} \alpha^{2}-9 v_{2} v_{1}^{2} \eta^{3 / 2}-36 v_{2}^{2} \sqrt{\eta} v_{1}+36 v_{1}^{2} \sqrt{\eta} v_{2}+36 v_{2}^{2} \sqrt{\eta} \rho^{2}\right. \\
& \left.+72 v_{2}^{2} \sqrt{\eta} \rho p \alpha+36 v_{2}^{2} \sqrt{\eta} p^{2} \alpha^{2}-36 v_{1}^{2} \sqrt{\eta} p^{2}-32 \lambda \sqrt{3} p^{2} \rho v_{1} \sqrt{v_{2}}-32 \lambda \sqrt{3} p^{3} \alpha v_{1} \sqrt{v_{2}}\right),
\end{aligned}
$$

and $\hat{q}$ is related to the electric charge [see Eq. (43)].

In the usual analysis, one proceeds by taking the derivative of the functional $h$ with respect to the constants introduced in the Ansatz. These then yield the set of equations to be solved and the conserved charges $J$ and $Q$. However, in the presence of a Chern-Simons term in the action the analysis needs to be modified [30].

To obtain the near-horizon geometry we can directly solve the Einstein equations and the Maxwell equations. The set of Einstein equations is equivalent to taking

$$
\frac{\partial h}{\partial v_{1}}=0, \quad \frac{\partial h}{\partial v_{2}}=0, \quad \frac{\partial h}{\partial \eta}=0
$$

i.e., these equations continue to hold also for EMCS theory. Variations of the functional with respect to $p$, however, do not take into account the Chern-Simons contribution. Therefore the Maxwell equations must be used when $\lambda \neq 0$. Simply setting $\partial h / \partial p=0$ would lead to a wrong result in this case. 
JOSE LUIS BLÁZQUEZ-SALCEDO et al.

The Einstein equations then yield the following algebraic relations

$$
\begin{aligned}
v_{2} & =v_{1}, \\
\eta v_{1} & =-\frac{4}{3} \frac{(\rho-p+p \alpha)(\rho+p+p \alpha)}{\alpha^{2}-1}, \\
v_{1} & =\frac{2}{3} \frac{\alpha^{4} p^{2}-p^{2}+2 \alpha^{3} \rho p-4 \rho p \alpha+\alpha^{2} \rho^{2}-2 \rho^{2}}{\alpha^{2}-1},
\end{aligned}
$$

and the Maxwell equations lead to

$$
\begin{gathered}
3 \alpha v_{2}^{5 / 2} \sqrt{\eta} \rho+3 \alpha^{2} v_{2}^{5 / 2} \sqrt{\eta} p-4 \lambda \sqrt{3} p v_{1} v_{2} \rho \\
-4 \lambda \sqrt{3} p^{2} v_{1} v_{2} \alpha-3 p v_{1}^{2} \sqrt{v}_{2} \sqrt{\eta}=0
\end{gathered}
$$

Note, that we now have four algebraic relations for six unknown constants. Thus we have two independent parameters. For those we may choose the angular momentum $J$ and the electric charge $Q$.

In the usual near-horizon formalism, the angular momentum $J$ is obtained by taking the derivative of the action functional $h$ with respect to the associated constant $\alpha$, and the electric charge $Q$ is obtained by taking the derivative with respect to $\rho$. To see where modifications arise because of the CS term we now recall the expressions for the angular momentum and the electric charge as Noether charges [30-33].

The Noether charges can be obtained by integrating the corresponding charge densities [30]

$$
\begin{aligned}
Q_{\xi}^{\alpha \mu}= & -\left[\sqrt{-g}\left(\nabla^{\alpha} \xi^{\mu}-\nabla^{\mu} \xi^{\alpha}\right)\right. \\
& \left.-4\left(\xi^{\tau} A_{\tau}\right)\left(\sqrt{-g} F^{\alpha \mu}+\frac{2 \lambda}{3 \sqrt{3}} \epsilon^{\alpha \mu \beta \nu \rho} A_{\beta} F_{\nu \rho}\right)\right]
\end{aligned}
$$

over the $S^{3}$-sphere. When taking the Killing vector $\eta_{(1)}$ or $\eta_{(2)}$, the angular momentum $J$ is obtained:

$$
\begin{aligned}
J= & \int d \theta d \varphi_{1} d \varphi_{2} Q_{\xi_{\varphi_{1}}}^{\mathrm{tr}} \\
= & 64 \pi^{2} \frac{v_{2}^{3 / 2}}{v_{1}} \sqrt{\eta} p(\rho+p \alpha)+16 \pi^{2} \frac{v_{2}^{5 / 2}}{v_{1}} \eta^{3 / 2} \alpha \\
& -\frac{256}{9} \sqrt{3} \pi^{2} p^{3} \lambda .
\end{aligned}
$$

Since the very same result is obtained from the equation

$$
\frac{\partial h}{\partial \alpha}=J
$$

the usual near-horizon relation remains valid for the angular momentum.
PHYSICAL REVIEW D 92, 044025 (2015)

However, this is not true for the electric charge $Q$. For $Q$ we need to consider the charge density [30]:

$$
Q_{M}^{\alpha \mu}=4\left[\sqrt{-g} F^{\alpha \mu}+\frac{\lambda}{\sqrt{3}} \epsilon^{\alpha \mu \beta \nu \rho} A_{\beta} F_{\nu \rho}\right] .
$$

When we integrate this charge density over the $S^{3}$-sphere we obtain the charge $Q$ :

$$
\begin{aligned}
Q & =\int d \theta d \varphi_{1} d \varphi_{2} Q_{M}^{\mathrm{tr}} \\
& =-64 \pi^{2} \frac{v_{2}^{3 / 2}}{v_{1}} \sqrt{\eta}(\rho+p \alpha)+\frac{128 \pi^{2} \sqrt{3}}{3} \lambda p^{2} .
\end{aligned}
$$

Note that this is equivalent to

$$
\frac{\partial h}{\partial \rho}=\hat{q}=-Q-\frac{128 \pi^{2} \sqrt{3}}{9} \lambda p^{2},
$$

and thus $\partial h / \partial \rho \neq-Q$, in contrast to a naive application of the entropy function formalism. Consequently, the extremization of the entropy functional must be appropriately modified to obtain the proper set of equations and charges.

We are also interested in obtaining expressions for the horizon properties. The horizon angular momentum can be calculated from the standard Komar formula

$$
\begin{aligned}
J_{\mathrm{H}} & =\int d \theta d \varphi_{1} d \varphi_{2}(-\sqrt{-g})\left(\nabla^{t} \xi^{r}-\nabla^{r} \xi^{t}\right) \\
& =16 \pi^{2} \frac{v_{2}^{5 / 2}}{v_{1}} \eta^{3 / 2} \alpha .
\end{aligned}
$$

Finally, for the horizon area we find the following expression:

$$
A_{\mathrm{H}}=\int d \theta d \varphi_{1} d \varphi_{2} \sqrt{\left|\operatorname{det}\left(g^{(3)}\right)\right|}=16 \pi^{2} v_{2}^{3 / 2} \sqrt{\eta} .
$$

Unfortunately, for a generic nonzero CS coupling constant $\lambda, \lambda \neq \lambda_{\mathrm{SG}}$, it is not possible to give an explicit expression for $S=A_{\mathrm{H}} / 4 G_{5}$ as a function of $Q$ and $J$. A straightforward numerical analysis of the algebraic relations reveals a rather complicated picture, with several branches of solutions. We are now going to describe several cases in detail.

\section{B. Einstein-Maxwell: $\lambda_{\mathrm{EM}}=\mathbf{0}$}

In the pure EM case the CS term vanishes, since $\lambda=0$, and the above relations reduce to those obtained in the usual entropy formalism. The EM case is interesting, since it allows for two branches of near-horizon solutions [17-19].

The near-horizon branch starting from the MP solution is given by 


$$
\begin{aligned}
v_{1} & =v_{2}, \\
\eta & =\frac{2\left(v_{2}-2 p^{2}\right)}{v_{2}}, \\
\rho & =0, \\
\alpha & =-1, \\
J & =32 \pi^{2} v_{2}^{3 / 2} \sqrt{2-4 p^{2} / v_{2}}, \\
Q & =64 \pi^{2} p \sqrt{v}_{2} \sqrt{2-4 p^{2} / v_{2}},
\end{aligned}
$$

where $J$ and $Q$ are the angular momentum and the electric charge, respectively. The horizon angular momentum $J_{\mathrm{H}}$ and horizon area $A_{\mathrm{H}}$ are given by

$$
\begin{aligned}
& J_{\mathrm{H}}=16 \pi^{2} v_{2}^{3 / 2}\left(2-4 p^{2} / v_{2}\right)^{3 / 2}, \\
& A_{\mathrm{H}}=16 \pi^{2} v_{2}^{3 / 2} \sqrt{2-4 p^{2} / v_{2}}=J / 2 .
\end{aligned}
$$

Hence, along the full MP branch, the entropy is always proportional to the angular momentum and independent of the charge.

A second near-horizon branch starts from the $\mathrm{RN}$ solution, and is given by

$$
\begin{aligned}
v_{1} & =v_{2}=\frac{4\left(\alpha^{2}+1\right) \rho^{2}}{3\left(\alpha^{2}-1\right)^{2}}, \\
\eta & =\frac{1}{\alpha^{2}+1}, \\
p & =-\frac{\rho \alpha}{\alpha^{2}-1}, \\
J & =\frac{512 \sqrt{3} \pi^{2} \rho^{3} \alpha}{9\left(\alpha^{2}-1\right)^{3}}, \\
Q & =\frac{128 \sqrt{3} \pi^{2} \rho^{2}}{3\left(\alpha^{2}-1\right)^{2}} .
\end{aligned}
$$

It has horizon angular momentum and horizon area

$$
\begin{aligned}
J_{\mathrm{H}} & =\frac{128 \sqrt{3} \pi^{2} \rho^{3} \alpha}{9\left(\alpha^{2}-1\right)^{3}}=\frac{J}{4}, \\
A_{\mathrm{H}} & =\frac{128 \sqrt{3} \pi^{2} \rho^{3}\left(\alpha^{2}+1\right)}{9\left(\alpha^{2}-1\right)^{3}} \\
& =\frac{\sqrt{2} \pi 3^{3 / 4}}{2} J^{2} Q^{-3 / 2}+\frac{3^{1 / 4} \sqrt{2}}{48 \pi} Q^{3 / 2} .
\end{aligned}
$$

Along this branch, the area is not proportional to the angular momentum. Instead, the horizon angular momentum is proportional to the total angular momentum. Interestingly, only parts of the near-horizon branches are also realized as global solutions [17,18].

\section{C. $D=5$ supergravity: $\lambda_{\mathrm{SG}}=1$}

In the case of $D=5$ supergravity, $\lambda_{\mathrm{SG}}=1$, the global solutions are known [22], with the BMPV solution representing the special case of supersymmetric and thus ergo-free solutions [20]. It is straightforward to obtain the near-horizon solutions in this case. They can be expressed as [30]

$$
\begin{aligned}
v_{1} & =v_{2}=\mu / 4, \\
\eta & =1-\frac{j^{2}}{\mu^{3}}, \\
\alpha & =\frac{j}{\sqrt{\mu^{3}-j^{2}}}, \\
\rho & =-\frac{\sqrt{3} \mu^{2}}{4 \sqrt{\mu^{3}-j^{2}}}, \\
p & =\frac{\sqrt{3}}{4} \frac{j}{\mu}, \\
J & =-4 \pi^{2} j, \\
Q & =8 \sqrt{3} \pi^{2} \mu,
\end{aligned}
$$

where the parameters $\mu$ and $j$ essentially describe the electric charge and the total angular momentum.

The horizon angular momentum and the horizon area are given by

$J_{\mathrm{H}}=\frac{2 \pi^{2} j}{\mu^{3}}\left(\mu^{3}-j^{2}\right)$,

$A_{\mathrm{H}}=2 \pi^{2} \sqrt{\left|\mu^{3}-j^{2}\right|}=\frac{\sqrt{2}}{48 \pi} \sqrt{\left|\sqrt{3} Q^{3}-288 \pi^{2} J^{2}\right|}$.

This shows that there are again two branches of nearhorizon solutions. The first branch has $J^{2}>-\frac{4}{3 \sqrt{3} \pi} Q^{3}$, while the second branch has $J^{2}<-\frac{4}{3 \sqrt{3} \pi} Q^{3}$. The second branch thus corresponds to the BMPV branch. In terms of the global solutions, this branch is ergoregion free with vanishing horizon angular velocity. In contrast, the first branch corresponds to the ordinary branch which possesses an ergoregion. At the matching point of both branches the area vanishes.

\section{Generic values of $\lambda$}

Let us now consider the near-horizon solutions for generic values of the CS coupling constant $\lambda$. Note that the solutions cannot be given explicitly, except for the previous two cases. Also, for $\lambda \neq 0$ the CS term breaks the charge reversal symmetry $Q \rightarrow-Q$ for spinning solutions. Therefore, we need to consider positive and negative $Q$ separately for finite values of $\lambda$.

Let us first consider near-horizon solutions with positive $Q$. When $\lambda$ is increased from zero, the branch structure of 


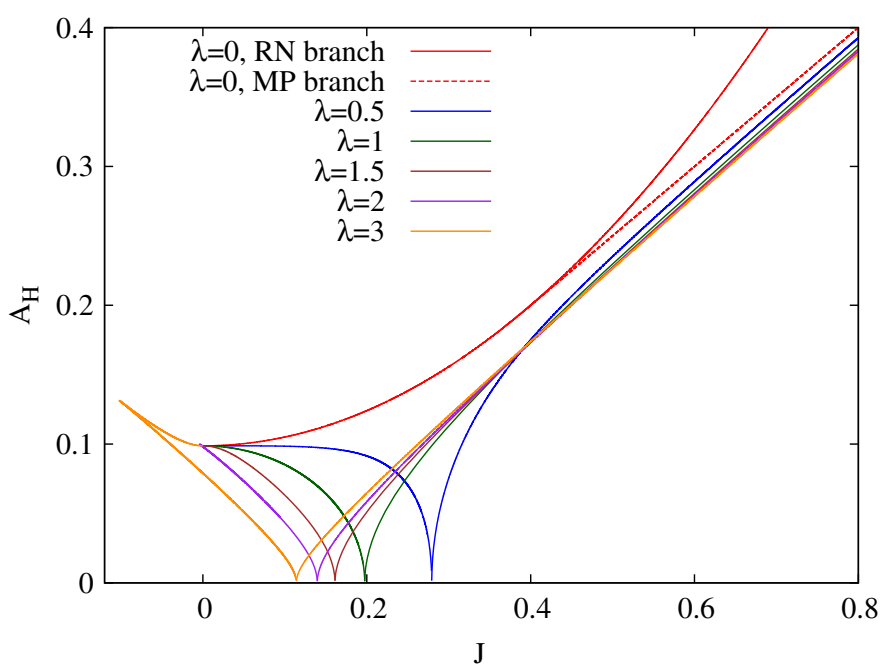

(a)

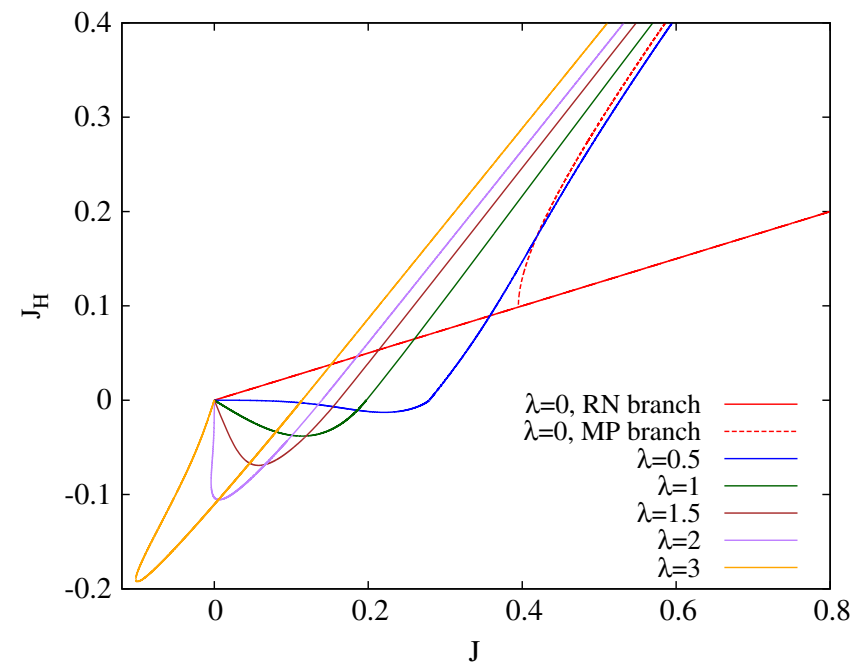

(b)

FIG. 1 (color online). Near-horizon solutions: (a) the horizon area $A_{\mathrm{H}}$ and (b) the horizon angular momentum $J_{\mathrm{H}}$ versus the angular momentum $J$ for CS coupling constant $\lambda=0,0.5,1,1.5,2$, and 3 and charge $Q=4$. The symmetric solutions obtained for $J \rightarrow-J$ are suppressed.

the pure EM case is lost. Instead a new branch structure emerges, which is generic for $0<\lambda<1.91$. It resembles the supergravity case, which is included as a particular case. After a small transition region $1.91<\lambda<2$ a new generic branch structure arises for $\lambda>2$.

Let us now demonstrate this $\lambda$-dependence in detail. In Fig. 1(a) we exhibit the horizon area $A_{\mathrm{H}}$ versus the angular momentum $J$ for several values of $\lambda$, where $\lambda$ is increased from 0 to 3 and the charge is fixed at the positive value $Q=4$. Note that the set of symmetric solutions obtained for $J \rightarrow-J$ is not exhibited here. Neither is it exhibited in Fig. 1(b), which shows the horizon angular momentum $J_{\mathrm{H}}$ versus $J$ for the same set of parameters.

Keeping in mind that a set of symmetric solutions is obtained for $J \rightarrow-J$, we focus our discussion for the moment on the solutions exhibited in the figure. For $\lambda \neq 0$ and positive $Q$, there exists always a solution with vanishing area. The angular momentum $J$ of this solution is finite. For fixed $Q$ its angular momentum decreases monotonically with increasing $\lambda$, as seen in Fig. 1(a). Figure 1(b) demonstrates that the horizon angular momentum changes smoothly at these solutions with vanishing area.

In the interval $0<\lambda<1.91$ there are two near-horizon branches. The small- $J$ branch extends from the extremal RN solution at $J=0$ to the solution with vanishing area, which has the maximal value of $J$ along this branch. The large- $J$ branch, on the other hand, extends from the solution with vanishing area and the minimal value of $J$ along this branch to solutions with arbitrarily large values of $J$ (Myers-Perry limit). Thus the structure of the near-horizon branches is essentially the same as the one found for the supersymmetric value $\lambda_{\mathrm{SG}}=1$, except that for $\lambda_{\mathrm{SG}}=1$ the small- $J$ branch is ergo-region free.
Let us next address the transition region $1.91<\lambda<2$. Here the branch structure changes. This is illustrated in Figs. 2(a) and 2(b). Note that the set of symmetric solutions obtained for $J \rightarrow-J$ is again not exhibited here to have more clarity in the figures.

When the horizon area $A_{\mathrm{H}}$ is considered as a function of the angular momentum $J$, as shown in Fig. 2(a), a swallowtail bifurcation is seen for any value of $\lambda$ in the transition region $1.91<\lambda<2$. Hence, there are solutions with the same value of $J$ but different values of the horizon area or the horizon angular momentum in the transition region. The reason for the emergence of the swallowtail bifurcation becomes clear by inspecting Fig. 2(b), where the angular momentum is shown versus the near-horizon parameter $v_{1}$. Here an inflection point arises at $\lambda=1.91$, and entails the occurrence of a local minimum and a local maximum for larger values of $\lambda$.

With increasing $\lambda$ the end points of the two cusps of the swallowtail bifurcation move to smaller values of $J$ and separate from each other. This has two interesting effects. The first effect is that, at the particular value of $\lambda=1.9425$, the upper cusp end point reaches the value $J=0$. The associated black hole solution thus corresponds to an extremal stationary black hole, that is not static; i.e., it differs from the extremal static Reissner-Nordström solution that is also present. (Of course, by the symmetry $J \rightarrow-J$ there are two such stationary nonstatic solutions. These have the same area but differ in the signs of their horizon angular velocities and horizon angular momenta.)

As seen in Fig. 2(a), for $1.9425<\lambda<2$ the upper cusp end point is at negative values of $J$ and thus the number of nonstatic $J=0$ solutions is doubled. With increasing $\lambda$, the upper cusp end point continues to move to lower values of $J$, and so does the lower cusp end point. However, for any 


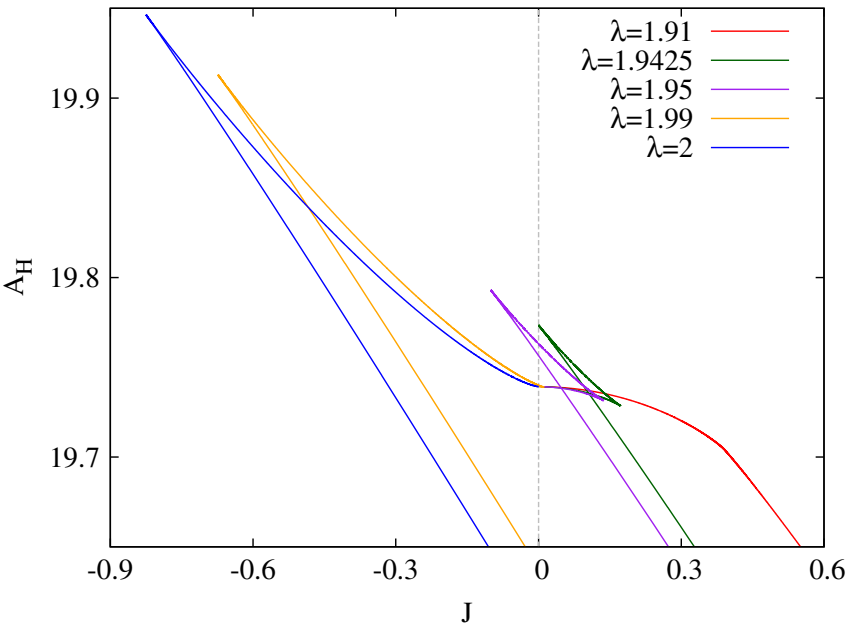

(a)

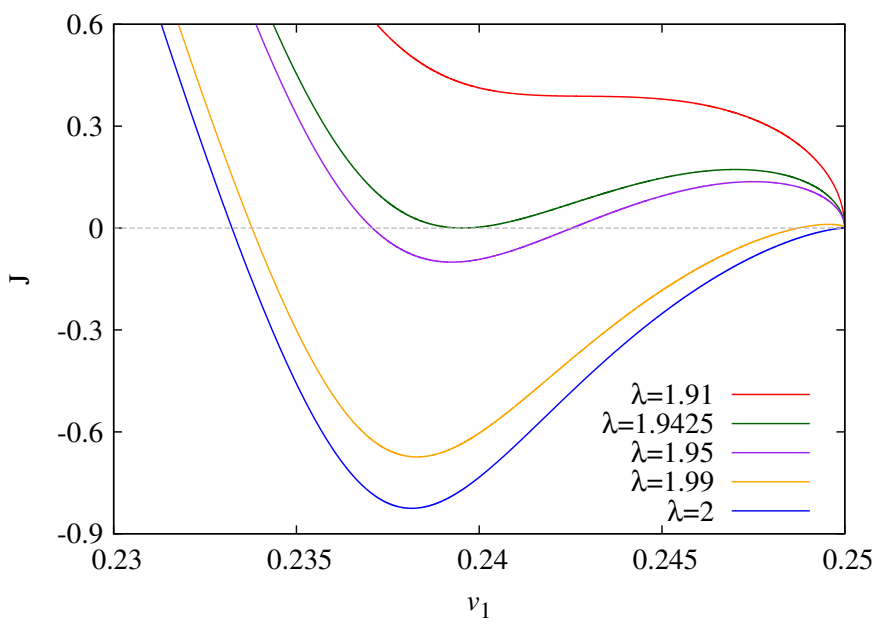

(b)

FIG. 2 (color online). Near-horizon solutions: (a) The area $A_{\mathrm{H}}$ versus the angular momentum $J$ for values of the CS coupling covering the vicinity of the transition region near the critical value $\lambda_{\text {cr }}=2$. The value of the charge is $Q=8 \sqrt{3} \pi^{2}$. The symmetric solutions for $J \rightarrow-J$ are not shown. (b) The angular momentum $J$ versus the parameter $v_{1}$ for the same sets of solutions.

value of $\lambda$, the extremal RN solution also resides at $J=0$, and is thus approached by the lower cusp end point. This leads to the second interesting effect, namely the disappearance of the lower cusp at the critical value $\lambda_{\mathrm{cr}}=2$, and thus the disappearance of one of these (two pairs of) $J=0$ solutions.

In particular, as seen in Fig. 2(b), when the critical value $\lambda_{\mathrm{cr}}=2$ is approached, the local minimum in $J$ deepens further, while the local maximum merges with the end point corresponding to the static solution. From the point of view of the full set of solutions, i.e., including the symmetric $J \rightarrow-J$ set of solutions, at the critical value $\lambda_{\text {cr }}=2$ two swallowtail bifurcations merge to form a single swallowtail bifurcation. Thus at $\lambda_{\mathrm{cr}}=2$ in total only one pair of nonstatic $J=0$ solutions remain together with the static RN solution.

Beyond the critical value $\lambda_{\mathrm{cr}}=2$ we observe a new generic branch structure, exhibited in Figs. 3(a) and 3(b). These figures show the full set of near-horizon solutions, including the symmetric set of solutions obtained for $J \rightarrow-J$ as well as the solutions for negative values of the charge. In the figures the value $\lambda=5$ is chosen for the CS coupling constant.

For positive $Q$ these near-horizon solutions possess three distinct branches, while they feature only a single branch for negative $Q$. Because of the symmetry $J \rightarrow-J$, all these branches have mirror branches in Fig. 3(a). Considering first positive $Q$, the small- $J$ branch starts from the extremal

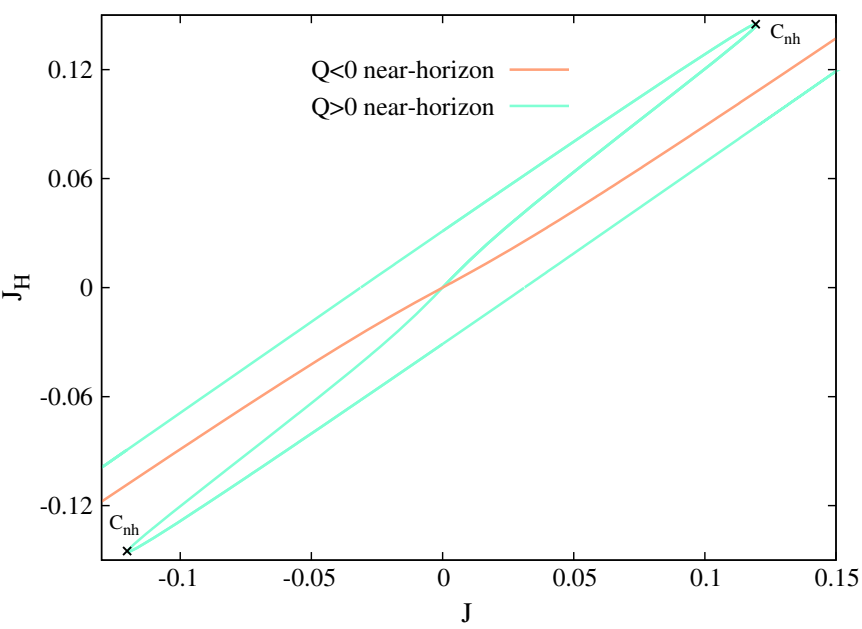

(b)

FIG. 3 (color online). Near-horizon solutions: (a) the horizon area $A_{\mathrm{H}}$ and (b) horizon angular momentum $J_{\mathrm{H}}$ versus the angular momentum $J$ for CS coupling constant $\lambda=5$. The value of the charge is $|Q|=1$. 
$\mathrm{RN}$ solution and extends until the cusp $C_{\mathrm{nh}}$ is reached. The intermediate- $J$ branch extends from the cusp $C_{\mathrm{nh}}$ to the solution with vanishing area. From there the large- $J$ branch extends towards solutions with arbitrarily large values of the angular momentum, reaching in the limit $J \rightarrow \infty$ the MP solution.

Clearly, beside the static Reissner-Nordstöm solution there are two symmetric $J=0$ solutions, which are nonstatic. As seen in Fig. 3(b) their horizon angular momenta are finite and opposite to each other. These nonstatic $J=0$ solutions are only present in the set of positive $Q$ solutions. Likewise the solutions with vanishing area are only present in the set of positive $Q$ solutions. In contrast, the negative $Q$ solutions show a rather unspectacular behavior. Their single branch connects directly from the RN solution to the MP solution for any finite value of the CS coupling constant $\lambda$.

In conclusion, we have seen that the branch structure of the near-horizon solutions depends significantly on the value of $\lambda$ and on the sign of the charge $Q$. In the next section we discuss the numerically obtained sets of global solutions and compare with the corresponding sets of nearhorizon solutions. We show that for negative $Q$, all near-horizon solutions correspond to global solutions. In contrast, when positive values of $Q$ are considered, not all of the near-horizon solutions are realized globally. Moreover, other near-horizon solutions may correspond to more than a single global solution.

\section{GLOBAL SOLUTIONS: NUMERICAL PROCEDURE}

Having obtained analytical expressions for the extremal solutions in the near-horizon formalism, we are now interested in obtaining global solutions, both extremal and nonextremal. As discussed in Sec. II, for generic values of the Chern-Simons coupling constant $\lambda$ and of the global charges, numerical integration of the differential equations seems necessary.

In the following we discuss the numerical procedure, the boundary conditions and the expansions of the functions employed to obtain generic EMCS black holes in five dimensions.

\section{A. Differential equations and boundary conditions}

Considering variation of the Lagrangian with respect to the $a_{0}(r)$ component of the gauge field we can obtain a first integral of the system in terms of the electric charge of the black hole:

$$
\begin{aligned}
\frac{d}{d r} a_{0}(r)= & -\frac{\omega(r)}{r} \frac{d}{d r} a_{\varphi}(r) \\
& +\frac{f(r)^{3 / 2}}{\sqrt{m(r) n(r)}}\left[\frac{4}{3} \sqrt{3} \lambda a_{\varphi}(r)^{2}-\frac{Q}{2}\right] .
\end{aligned}
$$

This differential equation is compatible with the Einstein and Maxwell equations. Combining this first-order differential equation with the system of differential equations, we obtain a minimal system of differential equations for the EMCS black hole solutions: four second-order differential equations for $f(r), m(r), \omega(r)$, and $a_{\varphi}(r)$; one first-order differential equation for $n(r)$; and one first-order differential equation for $a_{0}(r)$, which is decoupled from the other differential equations.

For the numerical calculations, we have found it useful to introduce a compactified radial coordinate. For the nonextremal solutions we take the compactified coordinate to be $x=1-r_{\mathrm{H}} / r$. In the extremal case we employ $x=\frac{r}{1+r}$. (Note that we are using an isotropic radial coordinate $r$, so $r_{\mathrm{H}}=0$ in the extremal case.) We employ a collocation method for boundary-value ordinary differential equations, equipped with an adaptive mesh selection procedure [34]. Typical mesh sizes include $10^{3}-10^{4}$ points. The solutions have a relative accuracy of $10^{-10}$. The estimates of the relative errors of the global charges and the magnetic moment are of order $10^{-6}$, giving rise to an estimate of the relative error of the gyromagnetic ratio $g$ of order $10^{-5}$.

To obtain asymptotically flat solutions the metric functions should satisfy the boundary conditions at infinity:

$$
\left.f\right|_{r=\infty}=\left.m\right|_{r=\infty}=\left.n\right|_{r=\infty}=1,\left.\quad \omega\right|_{r=\infty}=0 .
$$

For the gauge potential we choose a gauge in which it vanishes at infinity:

$$
\left.a_{0}\right|_{r=\infty}=\left.a_{\varphi}\right|_{r=\infty}=0 .
$$

Requiring the horizon to be regular, the metric functions must satisfy the boundary conditions

$$
\left.f\right|_{r=r_{\mathrm{H}}}=\left.m\right|_{r=r_{\mathrm{H}}}=\left.n\right|_{r=r_{\mathrm{H}}}=0,\left.\quad \omega\right|_{r=r_{\mathrm{H}}}=r_{\mathrm{H}} \Omega_{\mathrm{H}},
$$

where $\Omega_{\mathrm{H}}$ is the horizon angular velocity, Eq. (18). The gauge potential satisfies at the horizon the conditions (25)

$$
\left.\zeta^{\mu} A_{\mu}\right|_{r=r_{\mathrm{H}}}=\Phi_{\mathrm{H}}=\left.\left(a_{0}+\Omega_{\mathrm{H}} a_{\varphi}\right)\right|_{r=r_{\mathrm{H}}},\left.\quad \frac{d a_{\varphi}}{d r}\right|_{r=r_{\mathrm{H}}}=0,
$$

with the constant horizon electrostatic potential $\Phi_{\mathrm{H}}$.

\section{B. Expansions}

From the asymptotic expansion of the metric functions and the gauge field functions we can extract various parameters of the black hole: 


$$
\begin{gathered}
f \rightarrow 1-\frac{M}{6 \pi^{2} r^{2}}, \quad m \rightarrow 1-\frac{M}{12 \pi^{2} r^{2}}, \\
n \rightarrow 1-\frac{M}{12 \pi^{2} r^{2}}, \quad \omega \rightarrow \frac{J}{4 \pi^{2} r^{3}}, \\
a_{0} \rightarrow \frac{Q}{4 \pi^{2} r^{2}}, \quad a_{\varphi} \rightarrow-\frac{\mu_{\mathrm{mag}}}{4 \pi^{2} r^{2}},
\end{gathered}
$$

where $M$ is the mass, $J$ the angular momentum and $\mu_{\text {mag }}$ the magnetic moment. From these charges we can calculate the black hole gyromagnetic ratio $g=\frac{2 M \mu_{\text {mag }}}{Q J}$.

Next we consider the expansion of the functions near the horizon. First we present the expansion for nonextremal black holes. Once the previous conditions are imposed, it can be seen that

$$
\begin{aligned}
f(r) & =f_{2}\left(r-r_{\mathrm{H}}\right)^{2}+o\left(\left(r-r_{\mathrm{H}}\right)^{3}\right), \\
m(r) & =m_{2}\left(r-r_{\mathrm{H}}\right)^{2}+o\left(\left(r-r_{\mathrm{H}}\right)^{3}\right), \\
n(r) & =l_{2}\left(r-r_{\mathrm{H}}\right)^{2}+o\left(\left(r-r_{\mathrm{H}}\right)^{3}\right), \\
\omega(r) & =\Omega_{\mathrm{H}}+o\left(r-r_{\mathrm{H}}\right), \\
a_{0}(r) & =a_{0,0}+o\left(\left(r-r_{\mathrm{H}}\right)^{2}\right), \\
a_{\varphi}(r) & =a_{\varphi, 0}+o\left(\left(r-r_{\mathrm{H}}\right)^{2}\right) .
\end{aligned}
$$

These constants are implicitly related to three global charges $(M, Q$ and $J)$ in the nonextremal case.

In the case of extremal black holes, the radial dependence of the functions in the vicinity of the horizon changes as follows:

$$
\begin{aligned}
f(r) & =f_{4} r^{4}+f_{\alpha} r^{(\alpha+4)}+o\left(r^{6}\right), \\
m(r) & =m_{2} r^{2}+m_{\beta} r^{(\beta+2)}+o\left(r^{4}\right), \\
n(r) & =l_{2} r^{2}+l_{\gamma} r^{(\gamma+2)}+o\left(r^{4}\right), \\
\omega(r) & =\Omega_{\mathrm{H}} r+\omega_{2} r^{2}+o\left(r^{3}\right), \\
a_{0}(r) & =a_{0,0}+a_{0, \lambda} r^{\delta}+o\left(r^{2}\right), \\
a_{\varphi}(r) & =a_{\varphi, 0}+l_{k, \mu} r^{\mu}+o\left(r^{2}\right) .
\end{aligned}
$$

Since we are considering the extremal case, all these constants are implicitly related to two global charges ( $Q$ and $J$ for example). The coefficients $\alpha, \beta, \gamma, \lambda$ and $\mu$ can be noninteger. In fact we have

$$
\begin{array}{lll}
0<\alpha<2, & 0<\beta<2, & 0<\gamma<2, \\
0<\delta<2, & 0<\mu<2, & 0<\nu<2 .
\end{array}
$$

In the pure Einstein-Maxwell case $(\lambda=0)$ the expansion contains similarly noninteger exponents. This feature is found in both EM branches (i.e., in the MP branch and in the RN branch).

Thus the expansion at the horizon in general leads to noninteger exponents. We observe that these exponents $(\alpha, \beta, \gamma, \lambda$ and $\mu$ ) can become lower than 1 . This could lead to a divergence of the first derivative of a reparametrized function. Hence we have to be very careful with the reparametrization we use for the numerics. We reparametrize the functions in the following form:

$$
\begin{aligned}
\hat{m} & =m(r) \\
\hat{n} & =n(r) \\
\hat{f} & =f(r) / x^{2} \\
\hat{\omega} & =(1-x)^{-2} \omega(r) \\
\hat{a}_{\varphi} & =x^{2} a_{\varphi}(r) .
\end{aligned}
$$

We then integrate the functions $\hat{m}, \hat{n}, \hat{f}, \hat{\omega}$ and $\hat{a}_{\varphi}$. The advantage of using these reparametrized functions is the following. Since the exponents $(\alpha, \beta, \gamma, \lambda$ and $\mu)$ are always greater than 0 , and the order of the differential equations is lower than or equal to 2 , there is no problem with diverging functions or derivatives in the numerics.

Note that all the redefined functions except for $\hat{\omega}$ now start with an $x^{2}$-term in the compactified coordinate $x=r /(r+1)$. (The reparametrization of $\omega$ is not related to the expansion at the horizon. It is done in order to be able to fix the angular momentum by a boundary condition.)

The boundary conditions must guarantee that the functions satisfy the horizon expansion $\left(\hat{m}, \hat{n}, \hat{f}\right.$ and $\hat{a}_{\varphi}$ start as $\left.x^{2}\right)$. At the horizon, $x=0$, we can impose the angular velocity by making $\hat{\omega}^{\prime}(0)=\Omega_{\mathrm{H}}$. Note that here a prime indicates derivation with respect to the compactified coordinate $x$.

At $x=1$ we impose asymptotic flatness by requiring

$$
\begin{aligned}
\hat{m}(1) & =1 \\
\hat{n}(1) & =1 \\
\hat{f}(1) & =1 \\
\hat{\omega}(1) & =0 \\
\hat{a}_{\varphi}(1) & =0 .
\end{aligned}
$$

Alternatively, we can also impose the angular momentum by requiring $\hat{\omega}^{\prime}(1)=-J /\left(4 \pi^{2}\right)$.

In the system of equations we are using, the $a_{0}$ function was eliminated by introducing a first integral of the system in terms of the electric charge. Using these boundary conditions we can obtain configurations with selected pairs of values for $\left(Q, \Omega_{\mathrm{H}}\right)$ or $(Q, J)$ for a given value of $\lambda$. Once a configuration is obtained, we can compute the global charges.

From the asymptotic behavior of the functions at $x=1$ we extract the mass $M$, the angular momentum $J$, and the magnetic moment $\mu_{\mathrm{mag}}$. With these quantities we calculate the gyromagnetic factor $g$. From the behavior of the functions at the horizon $x=0$ we extract the horizon mass 
$M_{\mathrm{H}}$, the horizon angular momentum $J_{\mathrm{H}}$, the horizon area $A_{\mathrm{H}}$ and the horizon electrostatic potential $\Phi_{H}$. The Smarr formula is always satisfied within the precision of the black hole configurations computed.

\section{GLOBAL SOLUTIONS: NUMERICAL RESULTS}

We now discuss the global EMCS black hole solutions obtained by numerical integration as described above, with emphasis on extremal configurations. Here we focus on the most interesting case for the CS coupling constant $\lambda$, namely $\lambda>2$. We first discuss the relation between the near-horizon solutions and the global solutions, and exhibit the intriguing branch structure of the latter. Subsequently we address the sequence of $J=0$ solutions and reveal the node structure of the two functions $a_{\varphi}(r)$ and $\omega(r)$. Finally, we discuss the domain of existence of the global solutions.

Concerning the lower values of $\lambda$, let us remark that for $0<\lambda<1.91$ the global extremal solutions are in one to one correspondence to the near-horizon solutions, while in the transitions region $1.91<\lambda<2$ this does no longer seem to be the case. When approaching the RN solution for values of $\lambda$ in the transition region and positive values of the charge, the functions of the rotating global extremal solutions develop highly divergent derivatives. This forbids definite statements on the existence and properties of the numerically constructed global solutions in this area of parameter space. However, our analysis indicates that the rotating global extremal solutions are disconnected from the static extremal RN black hole in the transition region. In particular, we did not obtain any nonstatic extremal $J=0$ solutions for $\lambda<2$.

\section{A. Near-horizon versus global solutions: The branch structure of extremal black holes}

Let us now recall the near-horizon solutions and overlay the corresponding properties of the global solutions for direct comparison. In Fig. 4 we exhibit the horizon area $A_{\mathrm{H}}$ and the horizon angular momentum $J_{\mathrm{H}}$ versus the total angular momentum $J$ for both sets of solutions. For definiteness, we have chosen CS coupling $\lambda_{\mathrm{CS}}=5$ and charge $Q= \pm 1$. However, the pattern observed has the same structure for all values of the CS coupling constant $\lambda>2$ and values of the charge $Q$. The near-horizon solutions are marked by thick lines, while the global solutions are marked by thin lines in the figures.

Clearly, for the negative charge solutions, shown in red, both global and near-horizon solutions match. Thus in this case all near-horizon solutions are realized globally. However, for positive charge solutions, shown in green (near-horizon) and blue (global), the situation is very different. The branches of global solutions end in the cusps $C_{[1,2]}$, while the near-horizon solution branches extend further to $C_{\mathrm{nh}}$. Thus the near-horizon solutions between both cusps are not realized globally. Moreover, the full near-horizon branch connecting the cusp $C_{\mathrm{nh}}$ and the extremal RN solution, which is marked by an asterisk, is also not realized globally-with the exception of the RN solution itself, of course.

Therefore we conclude that the static extremal RN solution with $Q>0$ is rotationally isolated from the family of global stationary extremal solutions, when $\lambda>2$ (and presumably already for $\lambda>1.91$ ). The horizon area $A_{\mathrm{H}}$ of the global solutions with $J=0$ is significantly smaller than

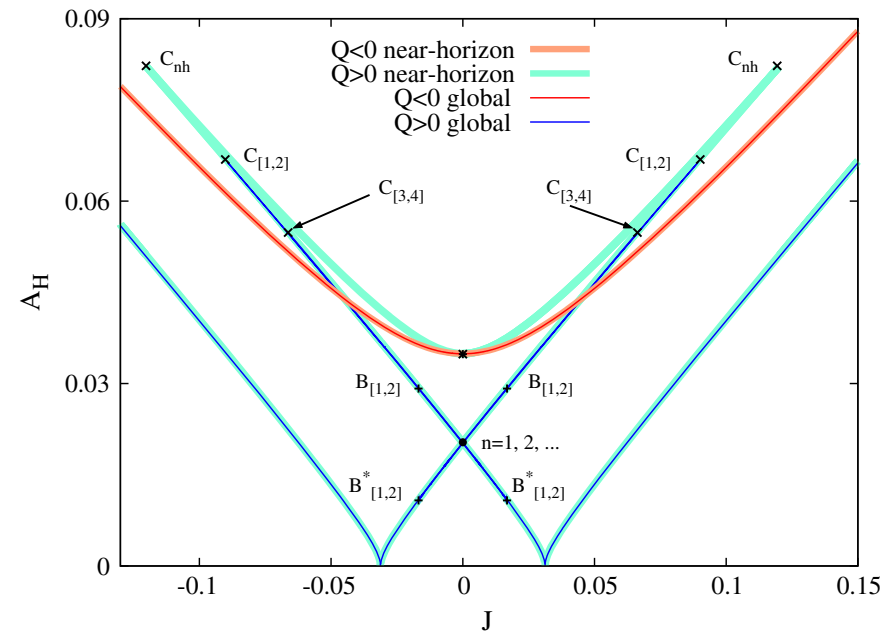

(a)

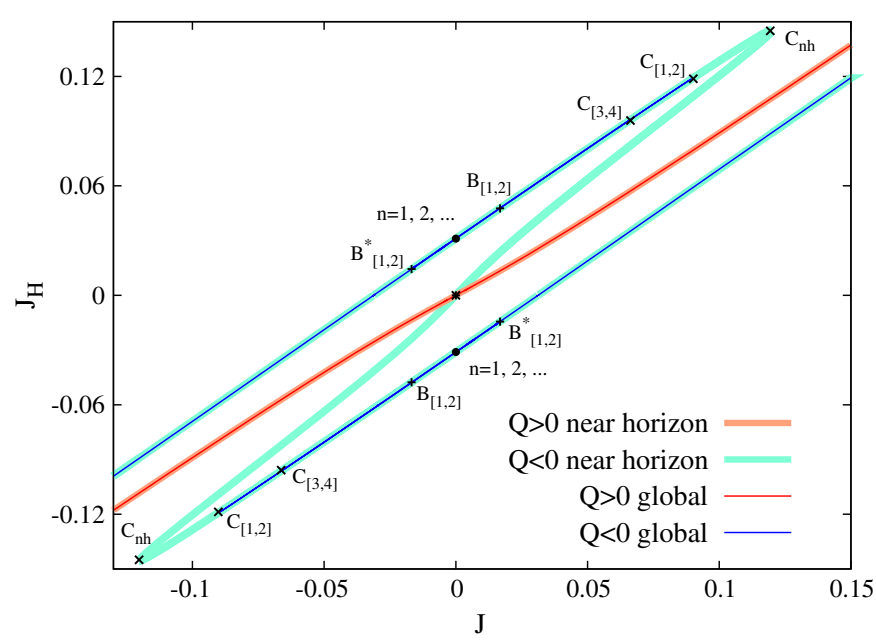

(b)

FIG. 4 (color online). Global versus near-horizon solutions: (a) the horizon area $A_{\mathrm{H}}$ and (b) the horizon angular momentum $J_{\mathrm{H}}$ versus the angular momentum $J$ for charge $|Q|=1$ and for CS coupling $\lambda=5$. The thick lines represent the near-horizon solutions for positive charge (green) and negative charge (red), while the thin lines represent the global solutions for positive charge (blue) and negative charge (red). The cusps are marked by $\times$ and denoted by $C_{[\ldots]}$; the bifurcation points are marked by + and denoted by $B_{[\ldots]}$ (see text). The black asterisk represents the extremal $\mathrm{RN}$ solution. 
the horizon area of the RN solution. Moreover, they carry finite horizon angular momentum $J_{\mathrm{H}}$.

We note that the existence of near-horizon solutions, which do not possess global counterparts, was noted first by Chen et al. [35] for the extremal dyonic black holes of $D=4$ Gauss-Bonnet gravity. However, in the EMCS case the relation between near-horizon solutions and global solutions is even more surprising, as discussed in the following. By considering the physical quantities, not accessible in the near-horizon formalism, like the mass $M$ or the horizon angular velocity $\Omega_{\mathrm{H}}$, we now present the intricate pattern of branches of the global rotating extremal black holes.

We exhibit in Fig. 5(a) the mass $M$ and in Fig. 5(b) the horizon angular velocity $\Omega_{\mathrm{H}}$ versus the total angular momentum $J$ of the global extremal black hole solutions. We have chosen CS coupling constant $\lambda=5$ and electric charge $Q= \pm 1$. These figures contrast the simple branch structure for negative charge (red) with the highly complicated branch structure for positive charge (blue).

Let us begin the detailed discussion of the branch structure for positive $Q$ with Fig. 5(a), which has a number of similar features to Fig. 4(a). For instance, the two points in Fig. 5(a), where a minimal value of the mass is encountered, correspond to the two singular solutions in Fig. 4(a), which have vanishing horizon area. We now choose one of these particular solutions, the one with $J<0$, as the starting point of our analysis of the branch structure.

When we decrease the angular momentum from this $J_{\mathrm{H}}<0, A_{\mathrm{H}}=0$ solution, we obtain a single infinite branch of extremal solutions, whose mass increases monotonically with decreasing $J$. In contrast, when we increase the angular momentum from this $J_{\mathrm{H}}<0, A_{\mathrm{H}}=0$ solution, we cross the first nonstatic $J=0$ solution, which we label

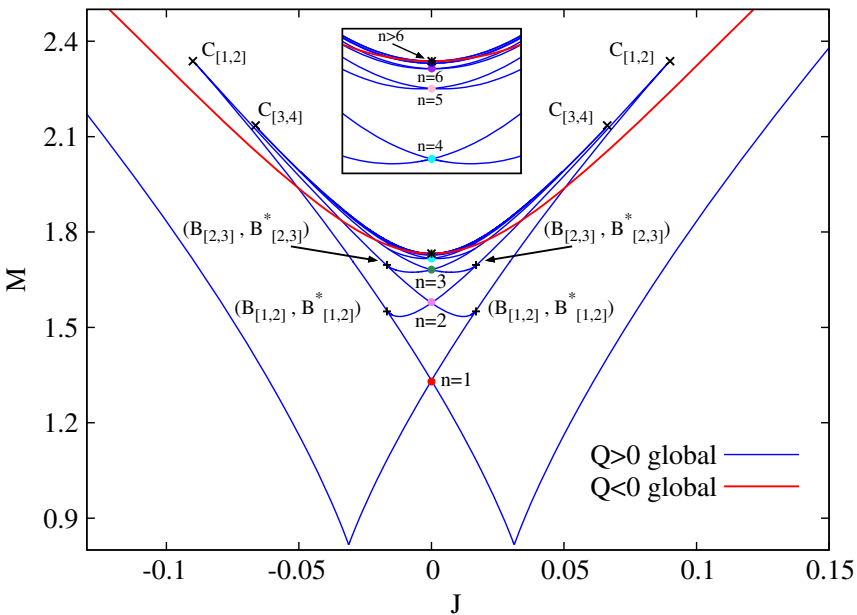

(a) $n=1$. Increasing $J$ further, we then cross a particular solution, which we label $B_{[1,2]}$. Eventually, this branch ends at a finite maximal value of $J$, where a cusp is encountered, which we label $C_{[1,2]}$. The solution at the cusp is perfectly regular.

From the cusp $C_{[1,2]}$ a new branch of solutions bends backwards towards smaller masses, as the angular momentum is decreased. Along this branch first another particular solution is encountered, which we label $B_{[2,3]}$. Then the branch crosses the next nonstatic $J=0$ solution, labeled $n=2$. It eventually ends in a solution labeled $B_{[1,2]}^{*}$, when it reaches the $J \rightarrow-J$ symmetric first branch at the solution $B_{[1,2]}$. Thus, we have encountered a bifurcation point, where the two branches meet, which has two distinct solutions, $B_{[1,2]}^{*}$ and $B_{[1,2]}$, that possess the same global charges.

This reveals the presence of nonuniqueness among the extremal solutions. While the global charges of these solutions are the same, and their horizon angular velocities are also the same, as seen in Fig. 5(b), they differ in their horizon area, as seen in Fig. 4(a). The area of $B_{[1,2]}^{*}$ is considerably smaller than the area of $B_{[1,2]}$.

These two sets of symmetric branches with cusps $C_{[1,2]}$ and bifurcation points $B_{[1,2]}, B_{[1,2]}^{*}$ form only the lowest mass solutions of a whole tower of branches, built in an analogous ever-repeating manner. We have already noted the presence of the particular (symmetric) points $B_{[2,3]}$. Here the next (symmetric) bifurcations are encountered, where new branches arise, whose initial solutions are labeled $B_{[2,3]}^{*}$.

Starting at the solution $B_{[2,3]}^{*}$ with negative $J$, we cross the next nonstatic $J=0$ solution, $n=3$. Increasing $J$

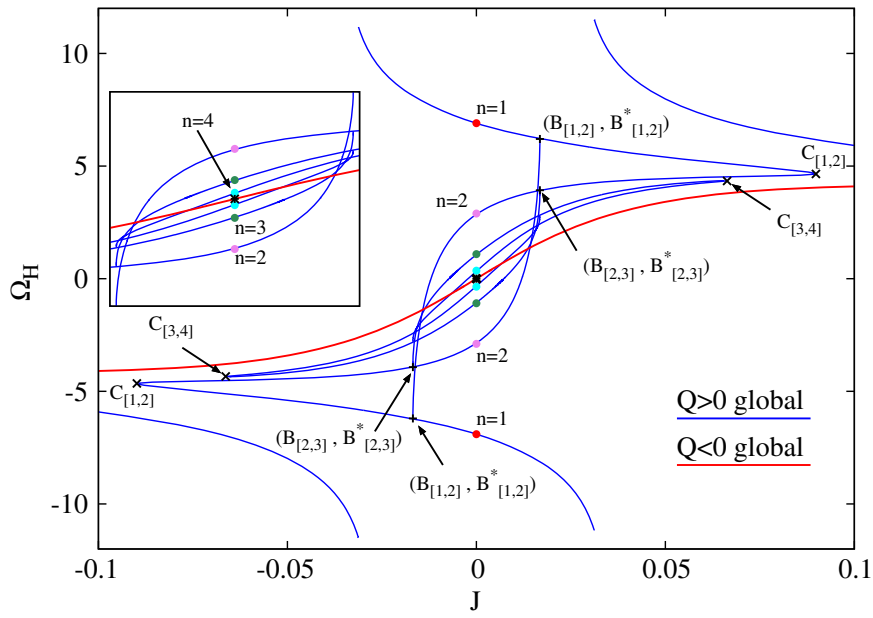

(b)

FIG. 5 (color online). Global solutions: (a) the total mass $M$ and (b) the horizon angular velocity $\Omega_{\mathrm{H}}$ versus the angular momentum $J$ for charge $Q=1$ (blue), $Q=-1$ (red) and for CS coupling $\lambda=5$. The cusps are marked by $\times$ and denoted by $C_{[\ldots]}$; the bifurcation points are marked by + and denoted by $B_{[\ldots]}$ (see text). The nonstatic $J=0$ solutions are numbered by $n$ and marked by red dots in different colors. The black asterisk represents the extremal RN solution. 


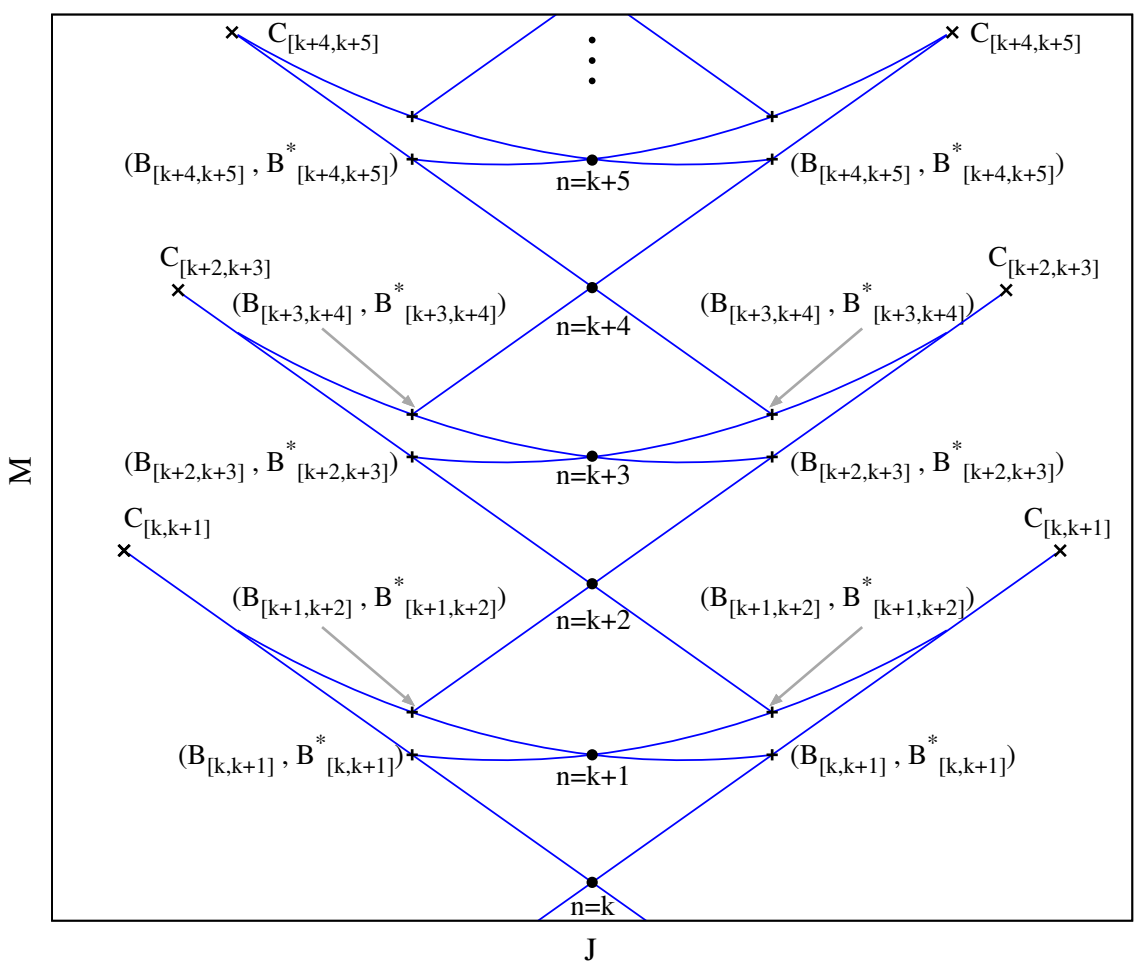

FIG. 6 (color online). Schematic representation of the branch structure of the global solutions: The total mass $M$ versus the angular momentum $J$. The cusps are marked by $\times$ and denoted by $C_{[\ldots]} ;$ the bifurcation points are marked by + and denoted by $B_{[\ldots]}$. The nonstatic $J=0$ solutions are numbered by $n$.

further, we then cross the solution at the next bifurcation point which we label $B_{[3,4]}$, and then encounter the next cusp $C_{[3,4]}$. From $C_{[3,4]}$ a new branch of solutions bends backwards towards smaller masses, as the angular momentum is decreased. Now the next bifurcation point is encountered, $B_{[4,5]}$; then the next nonstatic $J=0$ solution, $n=4$, is passed, and the branch ends at the bifurcation point, formed by $B_{[3,4]}^{*}$ and $B_{[3,4]}$.

This general scheme is exhibited schematically in Fig. 6. Starting from the solution $B_{[k+1, k+2]}^{*}$ with negative $J$, first the $(k+2)$ th nonstatic $J=0$ solution is encountered, then the bifurcation point $B_{[k+2, k+3]}$, and subsequently the cusp $C_{[k+2, k+3]}$. From $C_{[k+2, k+3]}$ a new branch bends backwards towards smaller masses, encountering first the bifurcation point $B_{[k+3, k+4]}$ and then the $(k+3)$ th nonstatic $J=0$ solution, and it ends at the bifurcation point, formed by $B_{[k+2, k+3]}^{*}$ and $B_{[k+2, k+3]}$.

For any $k$, the two solutions at the bifurcation points $B_{[k+2, k+3]}$ and $B_{[k+2, k+3]}^{*}$ possess the same global charges, whereas their horizon area and horizon angular momentum differ. Thus these solutions constitute a sequence of extremal solutions that violate uniqueness. The horizon angular velocities of the solutions $B_{[k+2, k+3]}$ and $B_{[k+2, k+3]}^{*}$, however, possess the same value. This is clearly seen for the lowest $k$ in Fig. 5(b). This figure also highlights the singular nature of the points with vanishing area, showing that the horizon angular velocity jumps from $\Omega_{\mathrm{H}}$ to $-\Omega_{\mathrm{H}}$ at these points.

Having generated in this way a whole sequence of branches, labeled by $n$, let us inspect the extent of these branches. Here we observe that with increasing $n$, the cusps $C_{n, n+1}$ occur at decreasing values of $|J|$. Thus the range of extremal global solutions for fixed $|J|$ decreases with increasing $n$.

Coming finally back to the comparison of global and near-horizon solutions, we conclude that a given nearhorizon solution can correspond to

(i) more than one global solution,

(ii) precisely one global solution,

(iii) no global solution at all.

In fact, we conjecture that a given near-horizon solution may even correspond to an infinite set of global solutions, as discussed in the following.

\section{B. Radial excitations: $\boldsymbol{J}=\mathbf{0}$ solutions}

Let us now address the question of how these branches of global extremal solutions differ. In particular, we would like to associate the integer $n$ counting the branches with a physical property of the solutions along these branches. To that end, let us first focus on the set of $J=0$ solutions. As seen in Fig. 5(a), the mass $M_{n}$ of each pair of degenerate $J=0$ solutions increases with $n$. This suggests that the solutions form a sequence of higher and higher excited 


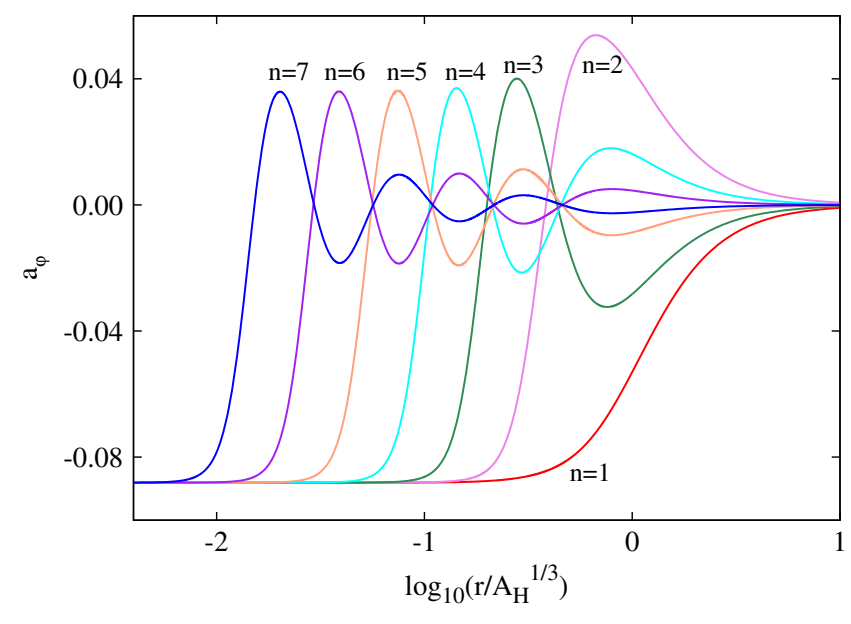

(a)

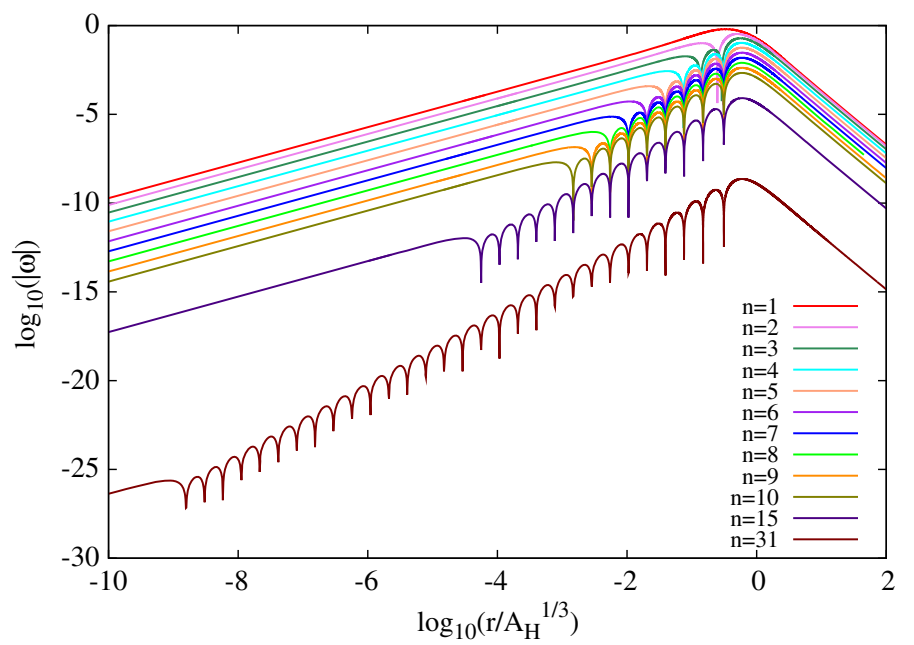

(b)

FIG. 7 (color online). Global solutions: (a) the gauge field function $a_{\varphi}$ and (b) the logarithm of the modulus of the metric function $\omega$ versus the logarithm of the scaled radial coordinate $\log _{10}\left(r / A_{\mathrm{H}}^{1 / 3}\right)$. The lowest radial excitations $n=1, \ldots, 7$ of $a_{\varphi}$ and $n=1, \ldots, 10,15$ and 31 of $\omega$ for charge $Q=1$ and CS coupling constant $\lambda=5$ are shown.

states, with the lowest mass solution forming the ground state or fundamental solution.

Excited states of otherwise very similar solutions are typically found when radial excitations are allowed. Examples range from the hydrogen atom, via sphaleron solutions in flat space [36], to hairy black holes [37], all featuring an infinite number of radial excitations of the ground state.

Indeed, it is the occurrence of radial excitations that results in the sequence of $J=0$ solutions and the associated intriguing pattern of branches of the global extremal black holes. We exhibit in Fig. 7(a) the gauge field function $a_{\varphi}$ and in 7(b) the metric function $\omega$ versus the radial coordinate. Again we have chosen charge $Q=1$ and CS coupling constant $\lambda=5$.

As the radial coordinate in the figure we have employed the logarithm of the scaled radial coordinate $\log _{10}\left(r / A_{\mathrm{H}}^{1 / 3}\right)$. Recall that all these solutions possess the same horizon area $A_{\mathrm{H}}$, as seen in Fig. 4(a). The first node always refers to spatial infinity. We note that in this logarithmic scale, the nodes of the solutions are located at roughly equal spacings. Moreover, the $k$ th nodes of the excited solutions with more than $k$ nodes are located very close to each other. (This is also known for the hydrogen atom and other radial excitations.)

For the metric function $\omega$ we have chosen to exhibit the logarithm of its modulus, since in contrast to $a_{\varphi}$ it varies over many orders of magnitude, as $n$ is increased. However, as seen in Fig. 7(b), beyond the nodes, for small values of the radial coordinate, the functions $\log _{10}|\omega|$ exhibit the same slope for all $n$. This also holds for large values of the radial coordinate. Besides the ten lowest $n$, Fig. 7 (b) also exhibits the metric function for $n=15$ and $n=31$. This clearly shows that the pattern continues to high values of $n$.
Solutions with $n$ in the thirties are the solutions with the highest node numbers we could achieve numerically so far. However, we conjecture that this sequence of $J=0$ solutions can be continued to arbitrarily high values of $n$. Thus we conjecture that we have obtained the lowest members of an infinite sequence of radially excited $J=0$ solutions. If this is really the case, then the corresponding near-horizon solutions will correspond to an infinite sequence of global solutions.

As mentioned already, with increasing node number $n$, the mass $M_{n}$ increases monotonically. In fact, it converges monotonically from below to the mass of the extremal static RN black hole, $M_{\mathrm{RN}}$. The Smarr formula then implies that the horizon electrostatic potential $\Phi_{n}$ converges likewise to the extremal static RN value, $\Phi_{\mathrm{RN}}$. At the same time, the horizon angular velocity $\Omega_{n}$ converges to zero, and thus also to the extremal static $\mathrm{RN}$ value. The horizon area $A_{\mathrm{H}}$, however, is independent of $n$ and has the same value for all the members of the (presumably) infinite sequence, and differs significantly from the extremal static $\mathrm{RN}$ value. The same holds true for the magnitude of the horizon angular momentum $J_{\mathrm{H}}$.

Let us address now the $\lambda$-dependence of the observed pattern of extremal $J=0$ solutions. As pointed out already, there is a lower critical value $\lambda_{\mathrm{cr}}$, beyond which no rotating $J=0$ solutions exist. When this critical value is approached from above, the mass of the rotating $J=0$ solutions should tend to the mass of the extremal static RN solution, in order to have a smooth emergence of these solutions at the critical value.

In Fig. 8 we demonstrate that this is indeed the case. Here we present the mass $M$ of the rotating $J=0$ solutions with charge $Q=1$ and up to seven nodes versus the CS 


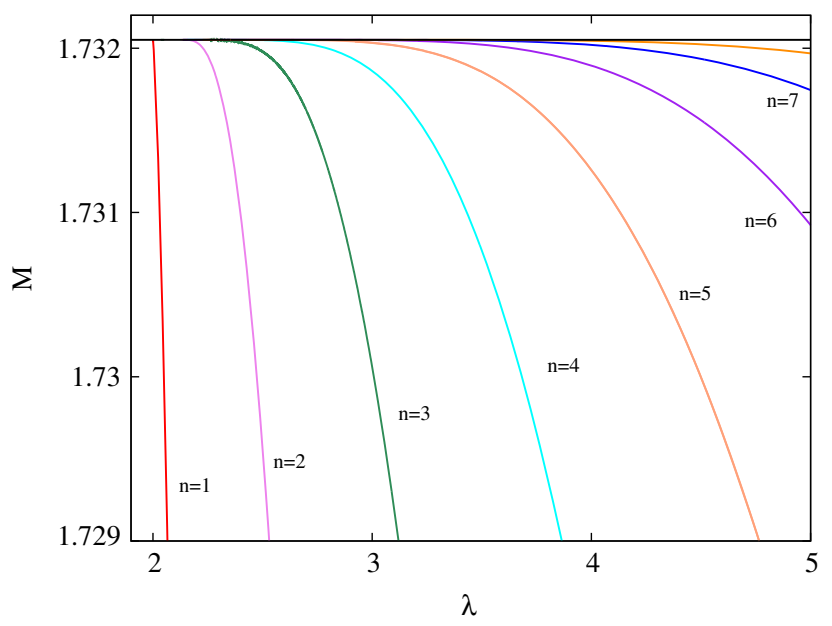

FIG. 8 (color online). Global solutions: The total mass $M$ versus the CS coupling constant $\lambda$. Shown are the lowest radial excitations $n=1, \ldots, 7$ for charge $Q=1$.

coupling $\lambda$. As expected the mass $M_{n}$ of the set of solutions with $n$ nodes approaches the mass of the static extremal RN solution $M_{\mathrm{RN}}$, when the CS coupling tends to $\lambda=2$. In particular, we observe that the larger the value of $n$, the closer is the mass $M_{n}$ to the limiting mass $M_{\mathrm{RN}}$ for fixed $\lambda$. To discern the branch structure, it is therefore preferable to employ values of $\lambda$ not too close to the critical value. This explains our choice $\lambda=5$ in most figures.

In Fig. 9 we demonstrate the sequence of $J=0$ solutions for a higher value of the CS coupling constant, $\lambda=10$. Obviously, the figures for the gauge function and the metric function are completely analogous to their counterparts, exhibited in Fig. 7.

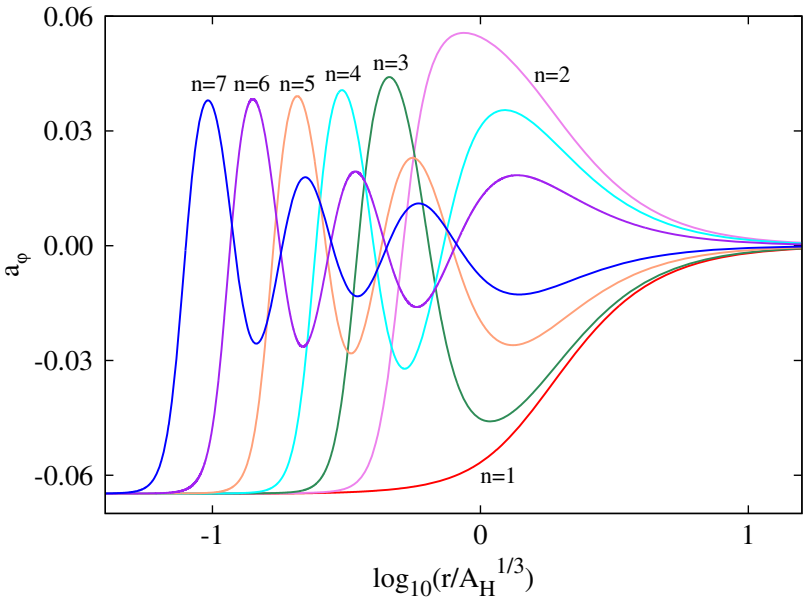

(a)
The nodal excitations seen in the sequence of $J=0$ solutions are also present in the general set of global extremal solutions. Along the various branches of solutions, new nodes appear from or disappear towards radial infinity. Thus all global extremal solutions possess an associated node number. However, the extent of the branches decreases with increasing $n$. Thus for a given $J \neq 0$, only a finite number of radial excitations may exist.

Likewise, also the nonextremal solutions possess an associated node number. Fixing, for instance, the horizon radius, families of nonextremal solutions are obtained that exhibit an intricate branch structure reminiscent of the one of the extremal solutions, but with fewer branches. However, the closer the family of nonextremal solutions approaches the set of extremal solutions, the more the number of branches increases.

Finally let us remark that the node number might possibly be considered in the quest to regain uniqueness.

\section{Domain of existence for $\lambda>2$}

Finally, we would like to address the domain of existence of global EMCS black hole solutions. Here we would like to first consider the global charges and, in particular, the angular momentum versus the charge for fixed mass. Typically, the boundary of the domain of existence of black hole solutions is then formed by extremal solutions, where the nonextremal solutions reside inside this boundary. Therefore we exhibit in Fig. 10(a) the scaled angular momentum $j=J / M^{3 / 2}$ versus the scaled charge $q=Q / M$ for extremal solutions, choosing again CS coupling $\lambda=5$. (A similar picture is found for other values of $\lambda>2$.) The figure includes the first few bifurcation points, cusps and nonstatic $J=0$ solutions. This allows us to connect the

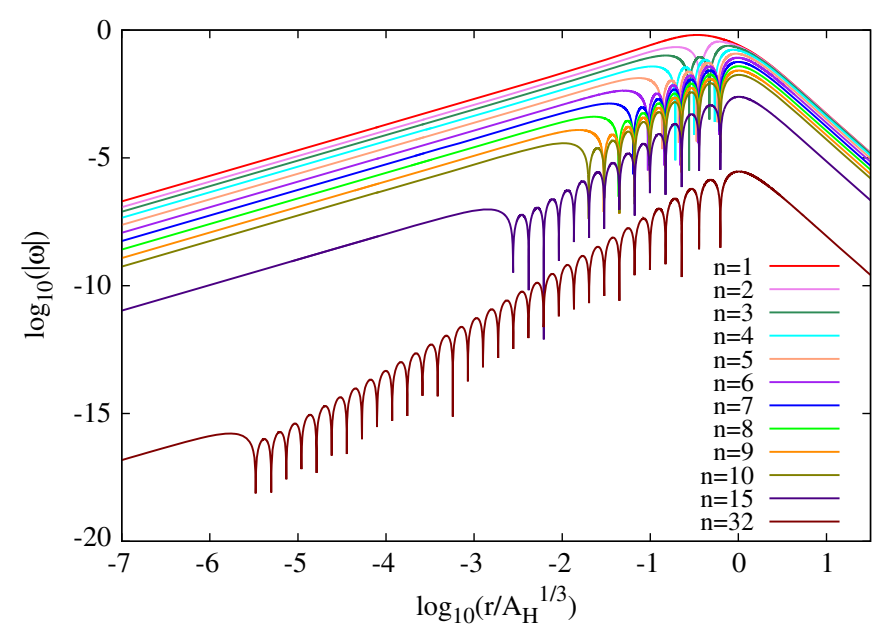

(b)

FIG. 9 (color online). Global solutions: (a) the gauge field function $a_{\varphi}$ and (b) the logarithm of the modulus of the metric function $\omega$ versus the logarithm of the scaled radial coordinate $\log _{10}\left(r / A_{\mathrm{H}}^{1 / 3}\right)$. The lowest radial excitations $n=1, \ldots, 7$ of $a_{\varphi}$ and $n=1, \ldots, 10,15$ and 32 of $\omega$ for charge $Q=1$ and CS coupling constant $\lambda=10$ are shown. 
locations of the solutions in the domain of existence with their locations in the previous figures.

Figure 10(a) then shows that when the scaled angular momentum is considered versus the scaled charge, the domain of existence is indeed delimited by branches of extremal solutions, which constitute its boundary. Clearly, the domain of existence is different for positive and negative charge solutions, since for spinning solutions the CS term breaks the charge reversal symmetry, $Q \rightarrow-Q$. The extremal solutions with negative charge form the smooth $Q<0$ boundary. This contains the extremal static RN solution, when the angular momentum vanishes. The extremal solutions with positive charge, on the other hand, reflect the complicated branch structure discussed above.

For positive charge only a subset of the extremal solutions forms the $Q>0$ boundary. This subset of

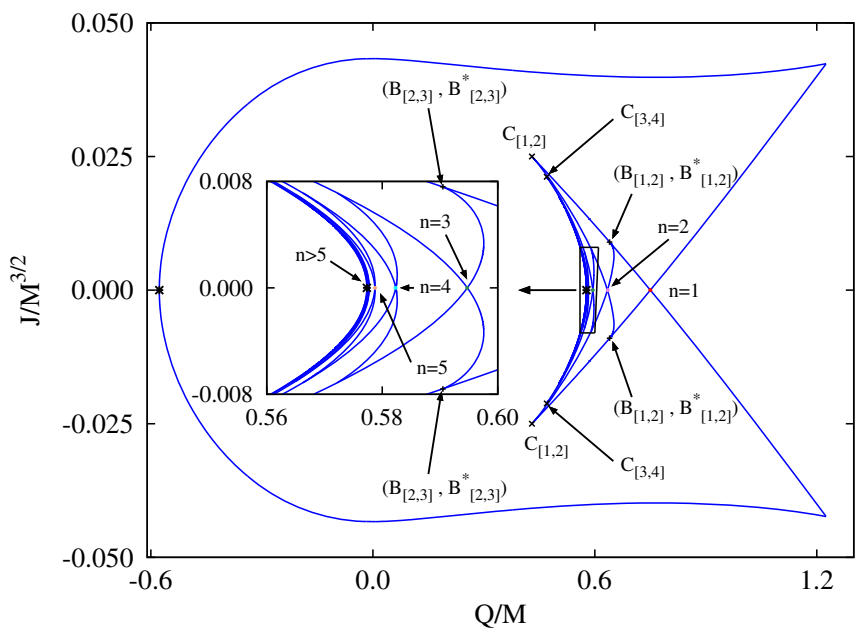

(a)

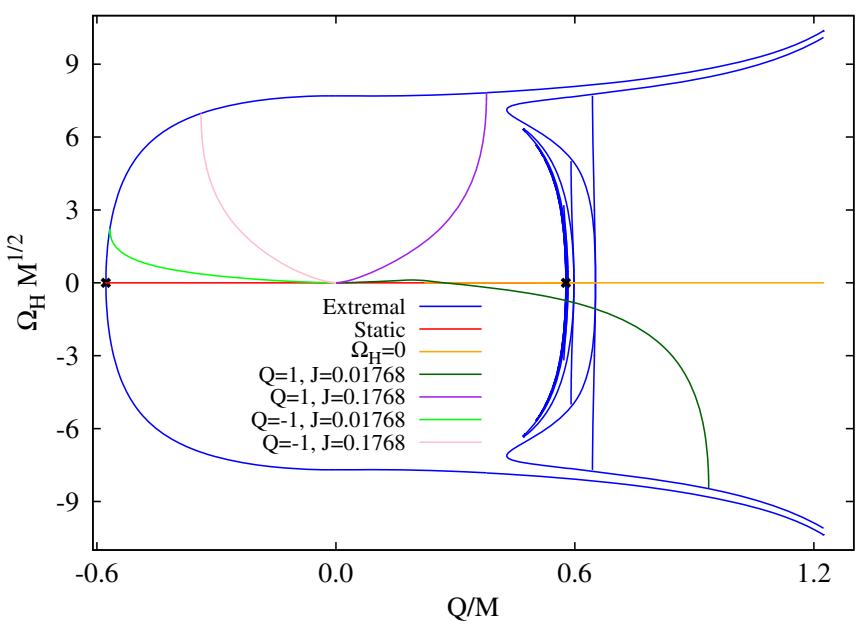

(c) solutions starts at the extremal Myers-Perry $(q=0)$ solutions, passes the cusps with vanishing horizon area, and ends at the first $(n=1)$ nonstatic $J=0$ solution. Thus in contrast to the $Q<0$ boundary, the $q>0$ boundary is not smooth, since it contains the pair of symmetric $J \rightarrow-J$ cusps, which reside at the maximal value $q_{\max }$ of the scaled charge $q$. The solutions at the cusps correspond to the singular solutions with vanishing horizon area in Fig. 4. Moreover, this maximal value of $q$ is larger than the value of $q$ of the corresponding extremal RN solution.

Even more surprising, however, is the fact that there are extremal solutions in the interior of the domain of existence. In particular, the $Q>0$ extremal RN solution is no longer part of the boundary. Such a feature does not occur in theories like pure Einstein-Maxwell theory, EinsteinMaxwell-dilaton theory, or EMCS theory for sufficiently low values of the CS coupling constant. This intriguing fact

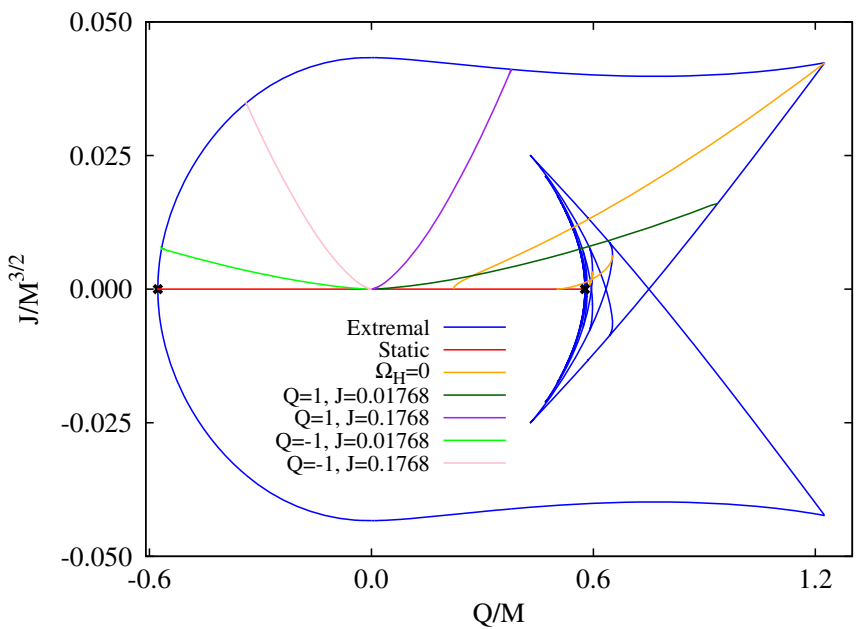

(b)

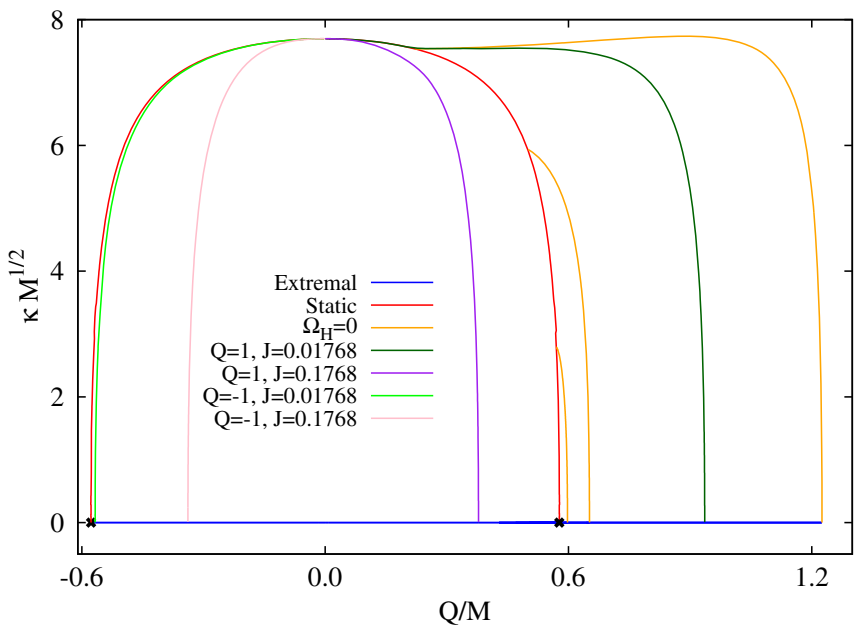

(d)

FIG. 10 (color online). Global and horizon properties: (a) the scaled angular momentum $j=J / M^{3 / 2}$ of extremal solutions including the bifurcation points and cusps versus the scaled charge $q=|Q| / M$; (b) the scaled angular momentum $j=J / M^{3 / 2}$ of extremal and selected sets of nonextremal solutions; (c) the scaled horizon angular velocity $\Omega_{\mathrm{H}} M^{1 / 2}$; and (d) the scaled surface gravity $\kappa M^{1 / 2}$ for the same sets of extremal and nonextremal solutions (CS coupling $\lambda=5$ ). 
produces a peculiar type of uniqueness violation: there exist both extremal and nonextremal solutions that possess the same global charges, $M, J$, and $Q$.

For more clarity and later reference, we replicate the demonstration of the domain of existence in Fig. 10(b), now without the inset, the bifurcations and the cusps, but instead with the inclusion of a number of selected sets of nonextremal solutions. These sets contain, in particular, the branches of nonstatic solutions with nonrotating horizon, $\Omega_{\mathrm{H}}=0$. Clearly, all solutions lie within the domain of existence, while the crossings of lines exhibit the nonuniqueness of the solutions.

Let us next consider the horizon properties of the solutions. In Fig. 10(c) we exhibit the scaled horizon angular velocity $\Omega_{\mathrm{H}} M^{1 / 2}$ for the extremal solutions and the same set of nonextremal solutions. Most of the boundary for the scaled horizon angular velocity $\Omega_{\mathrm{H}} M^{1 / 2}$ is formed by a subset of the extremal black holes; only the right-hand boundary, i.e., the large $q$ limit, is given by the maximum value of $q, q_{\max }$. All other extremal and all nonextremal solutions reside within these boundaries.

We exhibit the scaled surface gravity $\kappa M^{1 / 2}$ in Fig. 10(d). Clearly, the extremal black holes form the lower part of the boundary, since they all possess $\kappa=0$. For the $Q<0$ solutions, the static black holes form the upper part of the boundary, as is often the case. However, for the $Q<0$ solutions, the static solutions represent only the first part of the upper part of the boundary. Then another set of solutions adopts the role of forming the upper part of the boundary. Interestingly, this set corresponds precisely to the first set of nonstatic $\Omega_{\mathrm{H}}=0$ solutions, which start at a certain static RN solution and reach all the way to the singular cusp.

Let us next address the scaled area $a_{\mathrm{H}}=A_{\mathrm{H}} / M^{3 / 2}$. For $Q<0$, the extremal and the static solutions form again

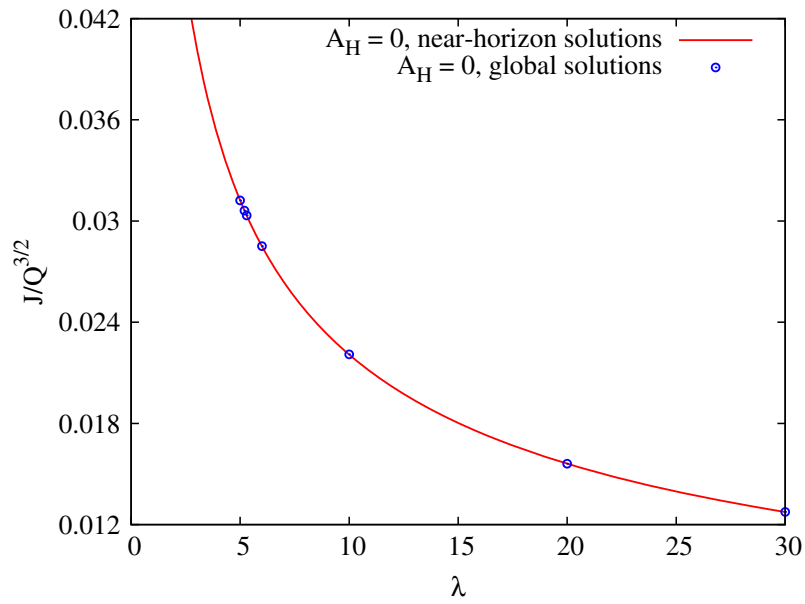

(a) together the boundary. For $Q>0$, the upper boundary part is also formed by the first part of the set of static solutions, until the first set of nonstatic $\Omega_{\mathrm{H}}=0$ solutions emerges from the static solutions, which then form the remaining upper part of the boundary.

Finally, we address the gyromagnetic ratio $g$ of the same sets of black hole solutions. Again, for $Q<0$, the gyromagnetic ratio is bounded by the static and extremal solutions. For $Q>0$, however, the gyromagnetic ratio becomes unbounded, as was noted before [23,24].

\section{CONCLUSIONS}

Here we have discussed global and near-horizon solutions of EMCS theory in five dimensions, focusing on solutions with equal magnitude angular momenta, to enhance the symmetry of the solutions, making the analytical and numerical analysis much more tractable while at the same time revealing already numerous intriguing features of the solutions.

Since the CS term breaks the charge reversal invariance, the families of solutions for negative charge and positive charge no longer agree when the CS term contributes, i.e., for rotating solutions. If we choose the CS coupling constant $\lambda$ to be positive, then the negative charge solutions do not represent any particular peculiarities. The positive charge solutions, however, develop a number of very interesting properties, as the CS coupling constant $\lambda$ first reaches the supergravity value $\lambda_{\mathrm{SG}}$ and then exceeds it.

At $\lambda_{\mathrm{SG}}$ the solutions are known analytically [22], with the BMPV solutions [20] forming a special case, which represent extremal nonstatic $\Omega_{\mathrm{H}}=0$ solutions, ending in a singular $A_{\mathrm{H}}=0$ solution. For $\lambda \neq \lambda_{\mathrm{SG}}$ the global solutions are known only perturbatively [11,13] or numerically [23-25], obtained by solving the corresponding set of

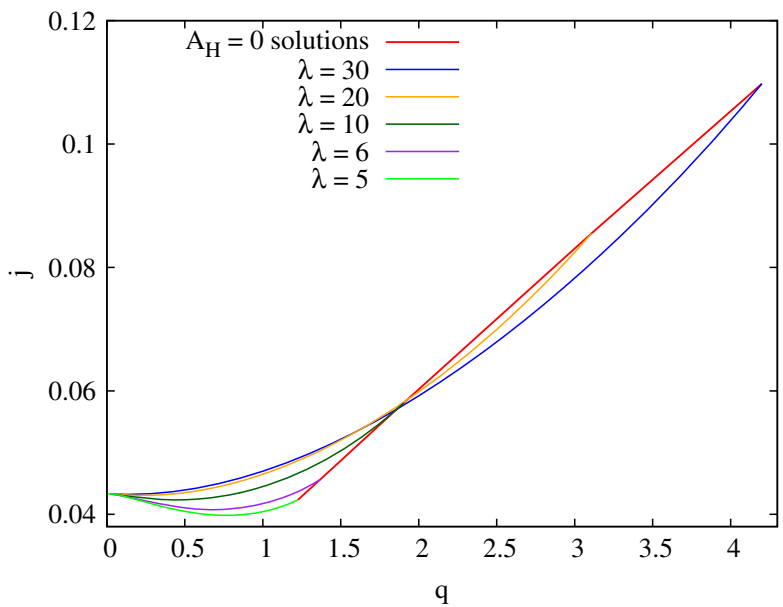

(b)

FIG. 11 (color online). Singular $A_{\mathrm{H}}=0$ solutions: (a) the scaled angular momentum $J / Q^{3 / 2}$ versus the CS coupling constant for nearhorizon and global solutions; (b) the scaled angular momentum $j=J / M^{3 / 2}$ versus the scaled charge $q=|Q| / M$ for global solutions (between the MP solution and the $A_{\mathrm{H}}=0$ cusp) and several values of $\lambda$. 
ordinary differential equations, subject to a set of appropriate boundary conditions. However, the near-horizon solutions can be obtained in a simpler way, by making use of an appropriately modified near-horizon formalism, where the modification is caused by the CS term.

The presence of a CS term always implies the occurrence of (a set of two degenerate $J \rightarrow-J$ symmetric) singular $A_{\mathrm{H}}=0$ solutions. But only for $\lambda \geq \lambda_{\mathrm{SG}}$ these singular solutions appear prominent in the domain of existence, residing at a cusp. We exhibit the $\lambda$-dependence of these solutions in Fig. 11. The cusp points have been obtained both for the near-horizon and the global solutions, as demonstrated in Fig. 11(a). When the cusp points are considered for the scaled angular momentum and the scaled charge, i.e., in the form $j(q)$, we note that they form an almost straight line, as depicted in Fig. 11(b).

Here we have focused our considerations on values of the CS coupling constant $\lambda>2 \lambda_{\mathrm{SG}}$, since above this limit a new type of extremal solution is present: extremal nonstatic $J=0$ solutions. These solutions come as degenerate pairs, symmetric with respect to $J \rightarrow-J$, where the lowest solution resides in the boundary of existence of the black hole solutions. The solutions form a presumably infinite sequence that can be labeled by an integer $n$. This integer is associated with the number of nodes of the metric function $\omega$ and the gauge field function $a_{\varphi}$. To our knowledge, this is the first example of black holes with Abelian fields which form excited states, which are reminiscent of the radial excitations of atoms, of sphalerons, or of hairy black holes.

With increasing node number the mass of these extremal nonstatic $J=0$ black holes converges to the mass of the extremal static RN black hole, while their horizon angular velocity tends to zero along with their magnetic moment. Their area, however, retains the same constant value, which is different from the static value. We demonstrate this pointwise convergence of the solutions in the Appendix.

The nonstatic $J=0$ solutions are located symmetrically within an intriguing pattern of branches of extremal solutions. Most of these branches of extremal solutions reside within the domain of existence of EMCS black holes, when the scaled angular momentum is considered versus the scaled charged. This is rather unusual, since the extremal solutions typically form the boundary of the domain of existence in this case. It has the interesting consequence that there is nonuniqueness between extremal and nonextremal black holes. Furthermore, there is nonuniqueness between extremal black holes that reside at the bifurcation points of the branches, while nonuniqueness between nonextremal black holes was reported already earlier [23].

The comparison between near-horizon solutions and global solutions has also led to surprises; namely we have seen that a given near horizon solution can correspond to (i) more than one global solution, possibly even an infinite set; (ii) precisely one global solution; or (iii) no global solution at all. Thus our findings show that the intuition based on known exact solutions cannot be safely applied in the general case. Moreover, the results of a near-horizon analysis of extremal solutions cannot be always safely extrapolated to the global case, as assumed sometimes in the literature.

Similar black holes can be obtained in EMCS theory in higher odd dimensions, when all the angular momenta have the same magnitude [24]. In particular, our preliminary results show that for $D=7,9$ this sequence of nonstatic $J=0$ solutions is also found, and the solutions can be characterized by the number of nodes of the corresponding metric and gauge field functions.

We conjecture that extremal black holes with similar properties may also exist in other theories, in particular, in an Einstein-Maxwell-dilaton theory in four dimensions [38]. In this case, however, a set of partial differential equations must be solved, making the corresponding analysis much more involved.

However, our next step will be the inclusion of a cosmological constant for these EMCS black holes. When the new intriguing phenomena observed for the asymptotically flat solutions survive in the presence of a negative cosmological constant, the AdS/CFT correspondence may imply interesting consequences for the associated four-dimensional field theories living on the boundary.

\section{ACKNOWLEDGMENTS}

We gratefully acknowledge support by the DFG Research Training Group 1620 "Models of Gravity" and by the Spanish Ministerio de Ciencia e Innovacion, research Project No. FIS2011-28013. The work of E. R. is supported by the FCT-IF programme and the CIDMA strategic Project No. UID/MAT/04106/2013.

\section{APPENDIX: SEQUENCES OF NONSTATIC $J=0$ EXTREMAL SOLUTIONS}

In this appendix we briefly illustrate the full set of functions and the dependence of the nodes for the sequence of nonstatic $J=0$ extremal solutions and compare with the corresponding $\mathrm{RN}$ functions.

We present in Fig. 12 the radial dependence of the functions of the fundamental $J=0$ solution and the first few excitations. We note that the functions $f, m, n$ and $a_{0}$ exhibit a fast convergence towards a limiting solution, which is represented by the static extremal RN solutions. However, this convergence is only local. At the horizon, $x=0$, the functions do not tend to the RN values in most cases. In the limit $n \rightarrow \infty$ the functions will then exhibit a jump. We notice this also for the function $a_{\varphi}$. Such a discontinuity is necessary to guarantee that these solutions, while approaching the corresponding $\mathrm{RN}$ solution, retain a horizon area which differs from the $\mathrm{RN}$ horizon area, but remains the same for all solutions of the sequence. 


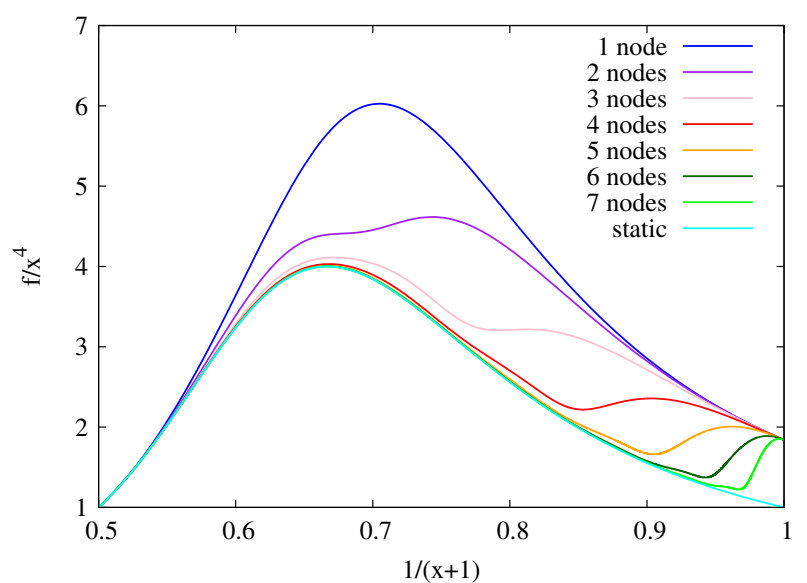

(a)

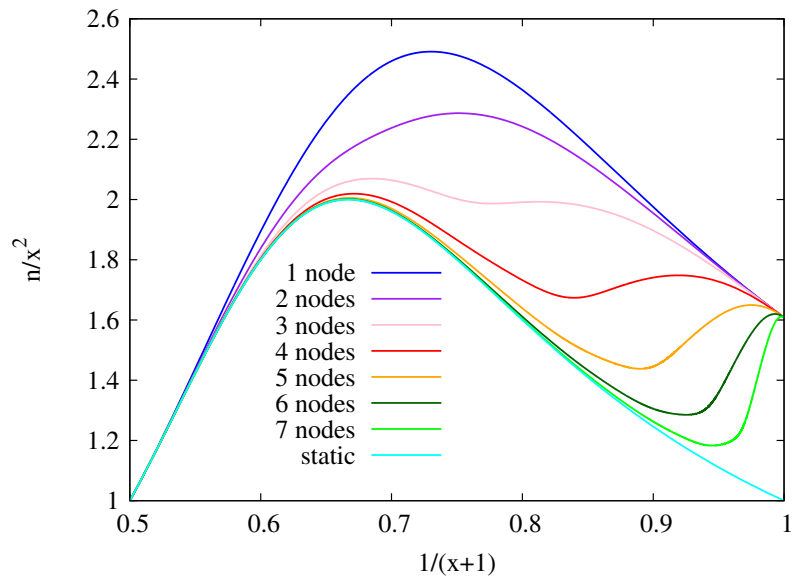

(c)

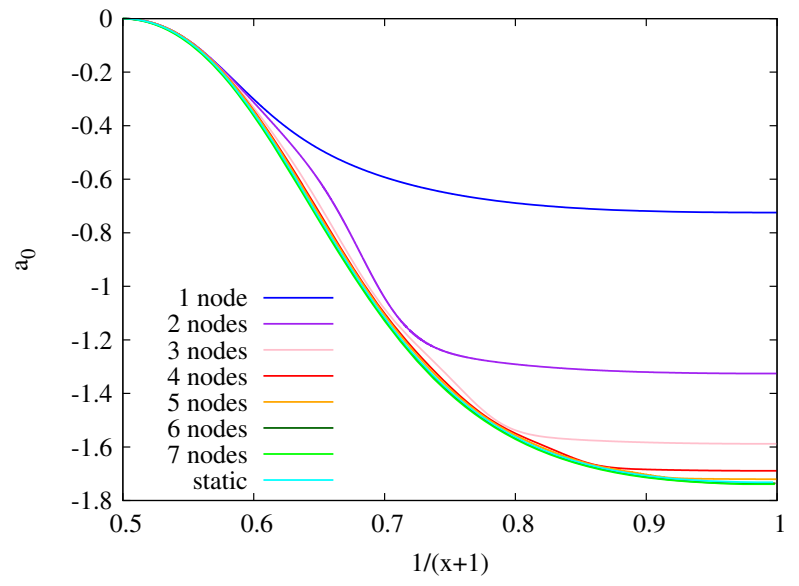

(e)

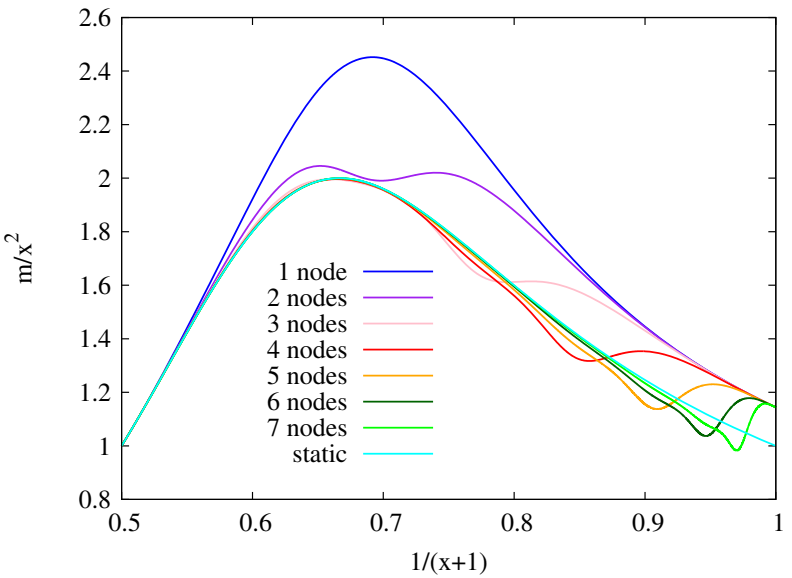

(b)

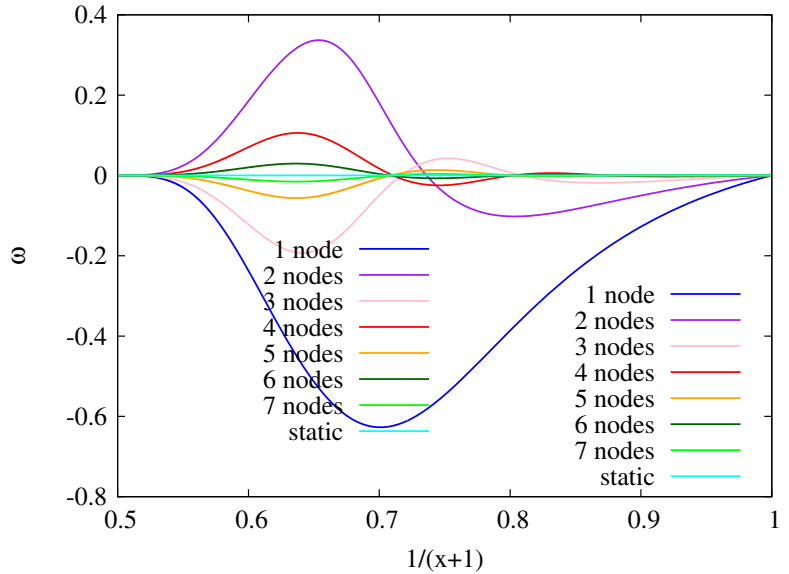

(d)

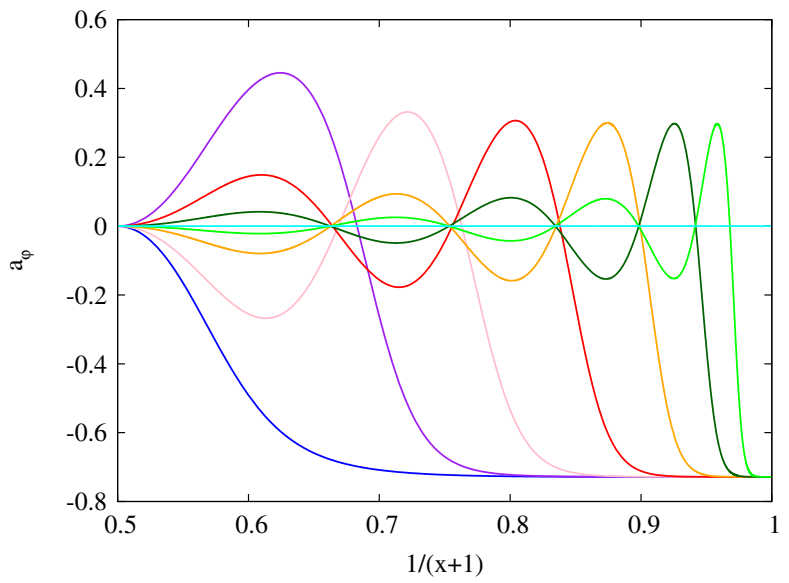

(f)

FIG. 12 (color online). Global $J=0$ solutions: The metric function (a) $f$, (b) $m$, (c) $n$, (d) $\omega$ and the gauge field functions (e) $a_{0}$ and (f) $a_{\varphi}$ versus the radial coordinate for the lowest radial excitations $n=1, \ldots, 7$. For comparison, the corresponding functions for the extremal RN solution are also shown. (Charge $Q=1$ and CS coupling $\lambda=5$.) 
[1] W. Israel, Event horizons in static electrovac space-times, Commun. Math. Phys. 8, 245 (1968).

[2] D. C. Robinson, Uniqueness of the Kerr Black Hole, Phys. Rev. Lett. 34, 905 (1975).

[3] P. O. Mazur, Proof of uniqueness of the Kerr-Newman black hole solution, J. Phys. A 15, 3173 (1982).

[4] P. T. Chrusciel, J. L. Costa, and M. Heusler, Stationary black holes: Uniqueness and beyond, Living Rev. Relativity 15, 7 (2012).

[5] R. C. Myers and M. J. Perry, Black holes in higher dimensional space-times, Ann. Phys. (N.Y.) 172, 304 (1986).

[6] R. C. Myers, Myers-Perry black holes, arXiv:1111.1903.

[7] A. N. Aliev and V. P. Frolov, Five dimensional rotating black hole in a uniform magnetic field: The gyromagnetic ratio, Phys. Rev. D 69, 084022 (2004).

[8] A. N. Aliev, Charged slowly rotating black holes in five dimensions, Mod. Phys. Lett. A 21, 751 (2006).

[9] A. N. Aliev, Rotating black holes in higher dimensional Einstein-Maxwell gravity, Phys. Rev. D 74, 024011 (2006).

[10] F. Navarro-Lérida, Perturbative charged rotating 5D Einstein-Maxwell black holes, Gen. Relativ. Gravit. 42, 2891 (2010).

[11] A. N. Aliev and D. K. Ciftci, A note on rotating charged black holes in Einstein-Maxwell-Chern-Simons theory, Phys. Rev. D 79, 044004 (2009).

[12] A. Sheykhi, M. Allahverdizadeh, Y. Bahrampour, and M. Rahnama, Asymptotically flat charged rotating dilaton black holes in higher dimensions, Phys. Lett. B 666, 82 (2008).

[13] M. Allahverdizadeh, J. Kunz, and F. Navarro-Lérida, Extremal charged rotating black holes in odd dimensions, Phys. Rev. D 82, 024030 (2010).

[14] M. Allahverdizadeh, J. Kunz, and F. Navarro-Lérida, Extremal charged rotating dilaton black holes in odd dimensions, Phys. Rev. D 82, 064034 (2010).

[15] J. Kunz, F. Navarro-Lérida, and A. K. Petersen, Fivedimensional charged rotating black holes, Phys. Lett. B 614, 104 (2005).

[16] J. Kunz, F. Navarro-Lérida, and J. Viebahn, Charged rotating black holes in odd dimensions, Phys. Lett. B 639, 362 (2006).

[17] J. L. Blázquez-Salcedo, J. Kunz, and F. Navarro-Lérida, Angular momentum-area proportionality of extremal charged black holes in odd dimensions, Phys. Lett. B 727, 340 (2013).

[18] J. L. Blázquez-Salcedo, J. Kunz, and F. Navarro-Lérida, Properties of rotating Einstein-Maxwell-Dilaton black holes in odd dimensions, Phys. Rev. D 89, 024038 (2014).

[19] H. K. Kunduri and J. Lucietti, Classification of near-horizon geometries of extremal black holes, Living Rev. Relativity 16, 8 (2013).
[20] J. C. Breckenridge, R. C. Myers, A. W. Peet, and C. Vafa, D-branes and spinning black holes, Phys. Lett. B 391, 93 (1997).

[21] M. Cvetic, H. Lu, and C. N. Pope, Charged Kerr-de Sitter black holes in five dimensions, Phys. Lett. B 598, 273 (2004).

[22] Z. W. Chong, M. Cvetic, H. Lu, and C. N. Pope, General Non-extremal Rotating Black Holes in Minimal FiveDimensional Gauged Supergravity, Phys. Rev. Lett. 95, 161301 (2005).

[23] J. Kunz and F. Navarro-Lérida, D = 5 Einstein-MaxwellChern-Simons Black Holes, Phys. Rev. Lett. 96, 081101 (2006).

[24] J. Kunz and F. Navarro-Lérida, Negative horizon mass for rotating black holes, Phys. Lett. B 643, 55 (2006).

[25] J. L. Blázquez-Salcedo, J. Kunz, F. Navarro-Lérida, and E. Radu, Sequences of Extremal Radially Excited Rotating Black Holes, Phys. Rev. Lett. 112, 011101 (2014).

[26] J. P. Gauntlett, R. C. Myers, and P. K. Townsend, Black holes of D $=5$ supergravity, Classical Quantum Gravity 16, 1 (1999).

[27] A. Sen, Black hole entropy function and the attractor mechanism in higher derivative gravity, J. High Energy Phys. 09 (2005) 038.

[28] D. Astefanesei, K. Goldstein, R. P. Jena, A. Sen, and S. P. Trivedi, Rotating attractors, J. High Energy Phys. 10 (2006) 058.

[29] K. Goldstein and R. P. Jena, One entropy function to rule them all, J. High Energy Phys. 11 (2007) 049.

[30] N. V. Suryanarayana and M. C. Wapler, Charges from attractors, Classical Quantum Gravity 24, 5047 (2007).

[31] R. M. Wald, Black hole entropy is the Noether charge, Phys. Rev. D 48, R3427 (1993).

[32] J. Lee and R. M. Wald, Local symmetries and constraints, J. Math. Phys. 31, 725 (1990).

[33] M. Rogatko, First law of black rings thermodynamics in higher dimensional Chern-Simons gravity, Phys. Rev. D 75, 024008 (2007).

[34] U. Ascher, J. Christiansen, and R. D. Russell, A collocation solver for mixed order systems of boundary value problems, Math. Comput. 33, 659 (1979); Collocation software for boundary-value ODEs, ACM Transactions on mathematical software (TOMS) 7, 209 (1981).

[35] C.-M. Chen, D. V. Gal'tsov, and D. G. Orlov, Extremal dyonic black holes in $\mathrm{D}=4$ Gauss-Bonnet gravity, Phys. Rev. D 78, 104013 (2008).

[36] J. Kunz and Y. Brihaye, New sphalerons in the WeinbergSalam theory, Phys. Lett. B 216, 353 (1989).

[37] M. S. Volkov and D. V. Galtsov, Black holes in Einstein Yang-Mills theory, Yad. Fiz. 51, 1171 (1990) [Sov. J. Nucl. Phys. 51, 747 (1990)].

[38] B. Kleihaus, J. Kunz, and F. Navarro-Lérida, Stationary black holes with static and counter rotating horizons, Phys. Rev. D 69, 081501 (2004). 